\title{
Symplectic and Poisson Geometry on Loop Spaces of Manifolds and Nonlinear Equations
}

\author{
Oleg Mokhov \\ Department of Geometry and Topology \\ Steklov Mathematical Institute \\ ul. Vavilova, 42 \\ Moscow, GSP-1, 117966, Russia \\ e-mail:mokhov@class.mian.su;mokhov@top.mian.su
}

We consider some differential geometric classes of local and nonlocal Poisson and symplectic structures on loop spaces of smooth manifolds which give natural Hamiltonian or multihamiltonian representations for some important nonlinear equations of mathematical physics and field theory such as nonlinear sigma models with torsion, degenerate Lagrangian systems of field theory, systems of hydrodynamic type, N-component systems of Heisenberg magnet type, Monge-Ampère equations, the Krichever-Novikov equation and others. In particular, complete classification of all nondegenerate Poisson bivectors $\omega^{i j}\left(x, u, u_{x}, u_{x x}, \ldots\right)$ depending on derivatives of the field variables $u^{i}(x)$ and the independent space variable $x$ is obtained $\left(u^{i}, i=1, \ldots, N\right.$, are local coordinates on smooth manifold $\left.M\right)$. In other words, all Poisson brackets of the following form

$$
\left\{u^{i}(x), u^{j}(y)\right\}=\omega^{i j}\left(x, u, u_{x}, u_{x x}, \ldots\right) \delta(x-y)
$$

$\operatorname{det}\left(\omega^{i j}\right) \neq 0$, are explicitly described. In addition, we shall prove integrability of some class of nonhomogeneous systems of hydrodynamic type and give a description of nonlinear partial differential equations of associativity in $2 D$ topological field theories (for some special type solutions of the Witten-Dijkgraaf-E.Verlinde-H.Verlinde (WDVV) system) as integrable nondiagonalizable weakly nonlinear homogeneous system of hydrodynamic type. 


\section{Generalized Poisson bivectors on loop spaces of manifolds}

Let be given an $N$-dimensional smooth manifold $M$ with local coordinates $\left\{u^{1}, \ldots, u^{N}\right\}$. Classic Poisson bivector $\omega^{i j}(u)$ on $M$ is by definition a skew-symmetric $(2,0)$-tensor on $M$ $\left(\omega^{i j}(u)=-\omega^{j i}(u)\right)$ which satisfies the well-known relation (the Jacobi identity):

$$
\frac{\partial \omega^{i j}}{\partial u^{s}} \omega^{s k}+\frac{\partial \omega^{j k}}{\partial u^{s}} \omega^{s i}+\frac{\partial \omega^{k i}}{\partial u^{s}} \omega^{s j}=0
$$

It gives a Poisson bracket on the space of smooth functions on $M$ :

$$
\{f(u), g(u)\}=\omega^{i j}(u) \frac{\partial f}{\partial u^{i}} \frac{\partial g}{\partial u^{j}}
$$

(or $\left\{u^{i}, u^{j}\right\}=\omega^{i j}(u)$ ) which is skew-symmetric

$$
\{f(u), g(u)\}=-\{g(u), f(u)\}
$$

and satisfies the Jacobi identity

$$
\{f(u),\{g(u), h(u)\}\}+\{g(u),\{h(u), f(u)\}\}+\{h(u),\{f(u), g(u)\}\}=0
$$

First of all, in this paper we would like to study some natural infinite-dimensional generalizations of the Poisson bivectors and the corresponding Poisson brackets.

Let us consider the loop space of the manifold $M$, i.e. the space $L(M)$ of all smooth parametrized mappings $\gamma: S^{1} \rightarrow M, \gamma(x)=\left\{u^{i}(x), x \in S^{1}\right\}$. The classic Poisson bivector $\omega^{i j}(u)$ gives so-called ultra-local Poisson bracket on the loop space $L(M)$ :

$$
\left\{u^{i}(x), u^{j}(y)\right\}=\omega^{i j}(u(x)) \delta(x-y)
$$

or, in other words, we have the Poisson bracket on the space of functionals on the loop space $L(M)$

$$
\{F, G\}=\int_{S^{1}} \frac{\delta F}{\delta u^{i}(x)} \omega^{i j}(u(x)) \frac{\delta G}{\delta u^{j}(x)} d x
$$

where $F$ and $G$ are arbitrary functionals on $L(M)$. In infinite-dimensional case of $L(M)$ we can consider a natural generalization of the Poisson bracket (1.4). In fact, generally speaking, in this case a Poisson bivector $\omega^{i j}$ can depend also on derivatives of the fields $u^{k}(x): \omega^{i j}\left(u, u_{x}, u_{x x}, \ldots\right)$.

Problem 1. Describe all Poisson brackets on $L(M)$ of the following form:

$$
\{F, G\}=\int_{S^{1}} \frac{\delta F}{\delta u^{i}(x)} \omega^{i j}\left(u, u_{x}, u_{x x}, \ldots\right) \frac{\delta G}{\delta u^{j}(x)} d x
$$

where $\operatorname{det}\left(\omega^{i j}\right) \neq 0$.

Remark 1.1. Note that in this paper we always consider only tensors depending on a finite number of derivatives of the fields $u^{k}(x)$.

Definition 1.1. We shall call a bivector $\omega^{i j}\left(u, u_{x}, u_{x x}, \ldots\right)$ by a generalized Poisson bivector on the loop space $L(M)$ if the tensor $\omega^{i j}\left(u, u_{x}, u_{x x}, \ldots\right)$ gives a Poisson bracket (1.5) on the space of functionals on $L(M)$. 
Theorem 1.1. Nondegenerate tensor $\omega^{i j}\left(u, u_{x}, u_{x x}, \ldots\right), \operatorname{det}\left(\omega^{i j}\right) \neq 0$, on the loop space $L(M)$ is a generalized Poisson bivector on $L(M)$ if and only if there are a closed 2-form $\Omega_{i j}(u)$ on $M(d \Omega=0)$ and a closed 3 -form $T_{i j k}(u)$ on $M(d T=0) \operatorname{such}$ that $\operatorname{det}\left(T_{i j k}(u) u_{x}^{k}+\right.$ $\left.\Omega_{i j}(u)\right) \neq 0$ and

$$
\omega^{i j}\left(T_{j k s}(u) u_{x}^{s}+\Omega_{j k}(u)\right)=\delta_{k}^{i}
$$

Remark 1.2. Thus, it follows from (1.6) that nondegenerate generalized Poisson bivectors $\omega^{i j}$ on $L(M)$ can depend only on the first derivatives of the field variables $u^{i}(x)$.

Remark 1.3. If the manifold $M$ is a symplectic one and the 2 -form $\Omega_{i j}(u)$ is a symplectic form on $M$ (i.e. $\operatorname{det}\left(\Omega_{i j}\right) \neq 0$ ) then for any closed 3 -form $T_{i j k}(u)$ on $M$ we have always that $\operatorname{det}\left(T_{i j k}(u) u_{x}^{k}+\Omega_{i j}(u)\right) \neq 0$ and the formula (1.6) gives explicitly a generalized Poisson bivector $\omega^{i j}$. We note also here that for any closed 3 -form $T_{i j k}$ on $M \operatorname{det}\left(T_{i j k}(u) u_{x}^{k}\right) \equiv 0$.

Theorem 1.1 describes translation invariant Poisson brackets but we can consider also Poisson bivectors $\omega^{i j}\left(x, u(x), u_{x}, u_{x x}, \ldots\right)$ depending explicitly on the independent space variable $x$.

Theorem 1.2. Tensor $\omega^{i j}\left(x, u, u_{x}, u_{x x}, \ldots\right), \operatorname{det}\left(\omega^{i j}\right) \neq 0$, gives a Poisson bracket

$$
\{F, G\}=\int_{S^{1}} \frac{\delta F}{\delta u^{i}(x)} \omega^{i j}\left(x, u, u_{x}, u_{x x}, \ldots\right) \frac{\delta G}{\delta u^{j}(x)} d x
$$

on the space of functionals on $L(M)$ if and only if there are an one-parameter family of 2 -forms $\Omega_{i j}(z, u)$ on the manifold $M$ and a closed 3 -form $R_{i j k}(u)$ on $M$ such that

$$
\operatorname{det}\left[\left(\int_{0}^{x}(d \Omega)_{i j k}(z, u(x)) d z+R_{i j k}(u)\right) u_{x}^{k}+\Omega_{i j}(x, u(x))\right] \neq 0
$$

and

$$
\omega^{i s}\left[\left(\int_{0}^{x}(d \Omega)_{s j k}(z, u(x)) d z+R_{s j k}(u)\right) u_{x}^{k}+\Omega_{s j}(x, u(x))\right]=\delta_{j}^{i},
$$

where

$$
(d \Omega)_{i j k}(z, u(x))=\frac{\partial \Omega_{i j}(z, u(x))}{\partial u^{k}}+\frac{\partial \Omega_{k i}(z, u(x))}{\partial u^{j}}+\frac{\partial \Omega_{j k}(z, u(x))}{\partial u^{i}}
$$

(note that there are no conditions of closure for the 2 -forms $\Omega_{i j}$ here as it takes place in the Theorem 1.1).

Remark 1.4. It is an interesting unsolved problem to classify all degenerate generalized Poisson bivectors $\omega^{i j}\left(x, u, u_{x}, u_{x x}, \ldots\right)$ with respect to dependence on derivatives of the fields $u^{i}(x)$.

In order that to prove the Theorems 1.1 and 1.2 we shall consider differential geometric objects which are inverse to nondegenerate Poisson bivectors $\omega^{i j}$. As it is well known, in finite-dimensional case we shall get symplectic forms $\omega_{i j}(u)$ on the manifold $M$ : $\omega_{i j}(u) \omega^{j k}(u)=\delta_{i}^{k},(d \omega)_{i j k}=0$. In the next section we shall consider corresponding generalizations of symplectic structures in connection with infinite-dimensional case of the loop space $L(M)$.

\section{Symplectic structures on loop spaces of manifolds}

The tangent space of the loop space $L(M)$ in its point (a loop) $\gamma$ consists of the all smooth vector fields $\xi^{i}, 1 \leq i \leq N$, defined along the loop $\gamma$. We shall denote it here by 
$T_{\gamma} L(M)$ (we note that $\xi^{i}(\gamma(x)) \in T_{\gamma(x)} M, \forall x \in S^{1}$, where $T_{\gamma(x)} M$ is the tangent space of the manifold $M$ in the point $\gamma(x))$.

Consider a skew-symmetric bilinear form $\omega(\xi, \eta)$ on the loop space $L(M)$ :

$$
\omega(\xi, \eta)=\int_{S^{1}} \xi^{i} A_{i j} \eta^{j} d x
$$

$\omega(\xi, \eta)=-\omega(\eta, \xi), \quad \xi, \eta \in T_{\gamma} L(M), \quad \xi=\left(\xi^{1}, \ldots, \xi^{N}\right), \quad \eta=\left(\eta^{1}, \ldots, \eta^{N}\right), \quad A=\left(A_{i j}\right)$ is a skew-symmetric operator defined by the loop $\gamma$

$$
A[\gamma]: T_{\gamma} L(M) \rightarrow T_{\gamma}^{*} L(M)
$$

Introduce a differential of 2-form $\omega(\xi, \eta)$ (it is an natural infinite-dimensional generalization of the usual differential in the Lie algebra of vector fields on a manifold):

$$
(d \omega)(\xi, \eta, \zeta)=\sum_{(\xi, \eta, \zeta)}\left\{\int_{S^{1}} \xi^{i} \frac{\delta \omega(\eta, \zeta)}{\delta u^{i}} d x+\omega(\xi,[\eta, \zeta])\right\}
$$

where $\xi, \eta, \zeta \in T_{\gamma} L(M)$,

$$
[\eta, \zeta]^{j}=\eta_{(k)}^{i} \frac{\partial \zeta^{j}}{\partial u_{(k)}^{i}}-\zeta_{(k)}^{i} \frac{\partial \eta^{j}}{\partial u_{(k)}^{i}}
$$

is a commutator of the vector fields $\eta$ and $\zeta, f_{(k)}=d^{k} f / d x^{k}$, and the sign $\sum_{(\xi, \eta, \zeta)}$ means that the sum is taken with respect to all cyclic permutations of the elements $(\xi, \eta, \zeta)$.

If $d \omega=0$, i.e. the 2 -form (2.1) is closed, then the operator $A_{i j}$ is called by a symplectic operator ( $\omega$ is called by presymplectic form or presymplectic structure).

Remark 2.1. In infinite-dimensional case presymplectic structure is often said to be a symplectic one even if the corresponding 2-form $\omega$ is degenerate on $L(M)$. We shall keep this terminology here (see [30] for refs. on symplectic operators and general theory of local Hamiltonian and symplectic structures and their connections with integrability of nonlinear partial differential equations).

Lemma 2.1. Nondegenerate tensor $\omega^{i j}\left(x, u, u_{x}, u_{x x}, \ldots\right), \operatorname{det} \omega^{i j} \neq 0$, is a generalized Poisson bivector on $L(M)$ if and only if the tensor $\omega_{i j}\left(x, u, u_{x}, u_{x x}, \ldots\right)$, where $\omega^{i s} \omega_{s j}=\delta_{j}^{i}$, is a symplectic operator.

It was shown by author in [13] that it is sufficient to verify the Jacobi identity only for all linear functionals of the form $F=\int_{S^{1}} f_{i}(x) u^{i}(x) d x$, where $f_{i}(x), i=1, \ldots, N$, are arbitrary functions. Consider vector fields $\xi$ of the form $\xi^{i}=\omega^{i j} f_{j}(x)$. It is easy to check that the condition

$$
(d \omega)(\xi, \eta, \zeta)=0
$$

for a symplectic structure $\omega_{i j}\left(x, u, u_{x}, u_{x x}, \ldots\right)$ is equivalent to the Jacobi identity on linear functionals for the bracket

$$
\left\{u^{i}(x), u^{j}(y)\right\}=\omega^{i j}\left(x, u, u_{x}, u_{x x}, \ldots\right) \delta(x-y) .
$$

Consider now symplectic operators $\omega_{i j}\left(u, u_{x}, u_{x x}, \ldots\right)$.

Lemma 2.2. An operator $\omega_{i j}\left(u, u_{x}, u_{x x}, \ldots\right)$ is a symplectic operator if and only if 1) $\omega_{i j}=-\omega_{j i}$ (skew-symmetry) 
2) for any vector-functions $\eta(x)=\left(\eta^{1}(x), \ldots, \eta^{N}(x)\right), \zeta(x)=\left(\zeta^{1}(x), \ldots, \zeta^{N}(x)\right)$ we have the following identity:

$$
(-1)^{k}\left(\frac{d}{d x}\right)^{k}\left(\frac{\partial \omega_{i j}}{\partial u_{(k)}^{s}} \eta^{i}(x) \zeta^{j}(x)\right)+\frac{\partial \omega_{s i}}{\partial u_{(k)}^{j}} \eta^{i}(x) \zeta_{(k)}^{j}(x)-\frac{\partial \omega_{s j}}{\partial u_{(k)}^{i}} \eta_{(k)}^{i}(x) \zeta^{j}(x) \equiv 0
$$

(the condition of closure for $\omega_{i j}$ ).

Consider now $R=\max \left\{m:\right.$ there exist $i, j, s$ such that $\left.\partial \omega_{i j} / \partial u_{(m)}^{s} \neq 0\right\}$.

If $R \geq 2$ then the coefficient at the term $\eta_{x}^{i}(x) \zeta_{(R-1)}^{j}(x)$ in $(2.3)$ is equal

$$
(-1)^{R} R \frac{\partial \omega_{i j}}{\partial u_{(R)}^{s}}
$$

i.e. $R \leq 1$ and the form $\omega_{i j}$ depends only on $u^{k}(x)$ and the first derivatives $u_{x}^{k}(x)$.

Now it is easy to show from the identity (2.3) that $\omega_{i j}$ must be quasilinear with respect to the first derivatives $u_{x}^{k}(x)$, i.e.

$$
\omega_{i j}\left(u, u_{x}\right)=T_{i j k}(u) u_{x}^{k}+\Omega_{i j}(u)
$$

Note that all symplectic operators of this type were described by author in [8] where differential geometric homogeneous symplectic operators of the first order

$$
A_{i j}=g_{i j} \frac{d}{d x}+b_{i j k}(u) u_{x}^{k}
$$

were studied in detail (after degeneration of $g_{i j}$ we get complete description of quasilinear symplectic structures $\left.\omega_{i j}\left(u, u_{x}\right)\right)$.

It turns out that symplectic operators $\omega_{i j}$ of the form (2.4) give Hamiltonian (or symplectic) structures of some special Lagrangian systems in classical field theory (some degeneration of nonlinear sigma models).

Theorem 2.1. Lagrangian systems $\delta S / \delta u=0$ that are given by the action

$$
S=\int\left(a_{i j}(u) u_{x}^{i} u_{t}^{j}+b_{i}(u) u_{t}^{i}+U\left(x, t, u, u_{x}, u_{x x}, \ldots\right)\right) d x d t
$$

with $b_{i}(u), U\left(x, t, u, u_{x}, u_{x x}, \ldots\right)$ being arbitrary functions and skew-symmetric functions $a_{i j}(u)\left(a_{i j}=-a_{j i}\right)$, are Hamiltonian systems with respect to the corresponding symplectic structures of the form (2.4).

Conversely, any Hamiltonian system of the form

$$
\left(T_{i j k}(u) u_{x}^{k}+\Omega_{i j}(u)\right) u_{t}^{j}=\frac{\delta U}{\delta u^{i}}
$$

is Lagrangian system with respect to the action of the type (2.5).

In the next section we shall consider symplectic geometry corresponding to the general nonlinear sigma models with torsion. 


\section{Nonlinear sigma models with torsion and symplectic geometry on loop spaces of manifolds}

Consider two-dimensional systems generated by the general nonlinear sigma model actions of the form

$$
S=\int\left(\frac{1}{2} r_{i j}(u) u_{x}^{i} u_{t}^{j}+U(u)\right) d x d t
$$

where $r_{i j}(u)$ is an arbitrary $(0,2)$-tensor and $U(u)$ is an arbitrary function on $M$. As it was shown by author in [8] the corresponding Lagrangian system

$$
\frac{\delta S}{\delta u}=0
$$

has always symplectic representation

$$
A_{i j} u_{t}^{j}=\frac{\delta U}{\delta u}
$$

where

$$
A_{i j}=\frac{1}{2}\left(r_{i j}+r_{j i}\right) \frac{d}{d x}+\frac{1}{2}\left(\frac{\partial r_{k i}}{\partial u^{j}}+\frac{\partial r_{i j}}{\partial u^{k}}-\frac{\partial r_{k j}}{\partial u^{i}}\right) u_{x}^{k}
$$

is a symplectic operator.

If $\operatorname{det}\left(r_{i j}+r_{j i}\right) \neq 0$ (in this case we have Riemannian or pseudoRiemannian manifold $M$ with the metric $\left.g_{i j}=\frac{1}{2}\left(r_{i j}+r_{j i}\right)\right)$ then the symplectic operator (3.3) gives a symplectic form $\omega(\xi, \eta)$ on $L(M)$ having the shape

$$
\omega(\xi, \eta)=\int_{\gamma}<\xi, \nabla_{\dot{\gamma}} \eta>
$$

where $\langle\xi, \eta\rangle=\frac{1}{2}\left(r_{i j}+r_{j i}\right) \xi^{i} \eta^{j}$ and $\nabla_{\dot{\gamma}}$ is a covariant derivation along $\gamma$ generated by some differential geometric connection $\Gamma_{j k}^{i}(u)$ on $M$. Let us describe all symplectic forms of the shape (3.4) on $L(M)$.

Theorem 3.1 [8]. Let be given a Riemannian or pseudoRiemannian manifold $\left(M, g_{i j}\right)$. A differential geometric connection $\Gamma_{j k}^{i}(u)$ on $M$ defines a symplectic form (3.4), where $<\xi, \eta>=g_{i j} \xi^{i} \eta^{j}$, if and only if

1) the connection $\Gamma_{j k}^{i}(u)$ is compatible with the metric $g_{i j}$, i.e.

$$
\nabla_{k} g_{i j} \equiv \frac{\partial g_{i j}}{\partial u^{k}}-g_{i s} \Gamma_{j k}^{s}-g_{j s} \Gamma_{i k}^{s}=0
$$

2) the torsion

$$
T_{i j k}=g_{i s} T_{j k}^{s}, T_{j k}^{i} \equiv \Gamma_{j k}^{i}-\Gamma_{k j}^{i}
$$

is a closed 3 -form on $M$.

Any closed 3-form on the (pseudo)Riemannian manifold $\left(M, g_{i j}\right)$ gives the only compatible with the metric $g_{i j}(u)$ differential geometric connection $\Gamma_{j k}^{i}(u)$ with torsion tensor determined explicitly by this closed 3-form and correspondingly any metric $g_{i j}(u)$ and any closed 3-form on $M$ generate a symplectic form (3.4) on $L(M)$. 
The corresponding symplectic operator has the form

$$
M_{i j}=g_{i j}(u) \frac{d}{d x}+g_{i s}(u) \Gamma_{j k}^{s}(u) u_{x}^{k}
$$

where locally we have

$$
\begin{gathered}
\Gamma_{j k}^{i}(u)=\frac{1}{2} g^{i s}(u)\left(\frac{\partial g_{s k}}{\partial u^{j}}+\frac{\partial g_{j s}}{\partial u^{k}}-\frac{\partial g_{j k}}{\partial u^{s}}+T_{s j k}(u)\right) \\
T_{i j k}(u)=\frac{1}{2}\left(\frac{\partial f_{i j}}{\partial u^{k}}+\frac{\partial f_{j k}}{\partial u^{i}}+\frac{\partial f_{k i}}{\partial u^{j}}\right) \\
f_{i j}(u)=-f_{j i}(u)
\end{gathered}
$$

Corollary. On any Riemannian or pseudoRiemannian manifold $\left(M, g_{i j}\right)$ closed 2-forms of the shape (3.4) on $L(M)$ are in one-to-one correspondence with closed 3 -forms on $M$.

An elementary conclusion is that on any two-dimensional Riemannian (or pseudoRiemannian) manifold $\left(M, g_{i j}\right)$ there is a single symplectic 2-form (3.4) on the loop space $L(M)$ and correspondingly a single symplectic operator (3.5) which are generated by the Levi-Civita connection. As for $N$-dimensional Riemannian (or pseudoRiemannian) manifolds, the conclusion is that there always exist an symplectic 2 -form of the shape (3.4) and a symplectic operator (3.5) which are generated by the Levi-Civita connection.

It must be noted that the symplectic form (3.4) is always degenerated (i.e. in fact, it is only a presymplectic form on $L(M))$ and the null space of $\omega(\xi, \eta)$ is constituted by vector fields parallel along $\gamma$. In particular, the velocity vector field $\left\{u_{x}^{i}\right\}$ belongs to the null space of $\omega(\xi, \eta)$ if $\gamma$ is a geodesic loop. Another subject is the interrelation between the symplectic forms under consideration and classic finite-dimensional symplectic structures on manifolds of geodesics. We remind that for $N$-dimensional Riemannian manifolds $\left(M, g_{i j}\right)$ whose geodesics are periodic and of equal length, there exists a $(2 N-2)$-dimensional symplectic manifold $C M$ of geodesics. The symplectic structure on $C M$ is determined by the curvature form of $S^{1}$-connection in the principal bundle $U M$ of unit tangent vectors of $\left(M, g_{i j}\right)$, where the $S^{1}$-connection is generated by the canonical 1-form on $T^{*} M$ (Reeb theorem, see [3336]). The tangent space $T_{\gamma} C M$ at the point $\gamma$ is isomorphic to the space of normal Jacobian fields along the geodesic $\gamma$.

Theorem 3.2 [8]. The restriction of the symplectic form (3.4) generated on $L(M)$ by the Levi-Civita connection to the finite-dimensional subspace of normal Jacobian fields along the geodesics $\gamma$ coincides with the symplectic Reeb form that is a closed nondegenerate 2-form on $C M$ defining a symplectic structure.

Theorem 3.3 [8]. Formulas (3.5)-(3.8) give complete description of all symplectic operators of the following shape

$$
\begin{gathered}
A_{i j}=a_{i j}(u) \frac{d}{d x}+b_{i j k}(u) u_{x}^{k}, \\
\operatorname{det} a_{i j}(u) \neq 0
\end{gathered}
$$

(homogeneous differential operators of the first order with nondegenerate higher coefficient). Lemma 3.1. An operator of the shape (3.9) is symplectic if and only if the following conditions are valid: 


$$
\begin{gathered}
a_{i j}=a_{j i} \\
\frac{\partial a_{i j}}{\partial u^{k}}=b_{i j k}+b_{j i k} \\
\frac{\partial a_{i j}}{\partial u^{k}}=b_{i k j}+b_{j k i} \\
\frac{\partial b_{j m k}}{\partial u^{i}}-\frac{\partial b_{j m i}}{\partial u^{k}}+\frac{\partial b_{i j k}}{\partial u^{m}}-\frac{\partial b_{i m k}}{\partial u^{j}}=0
\end{gathered}
$$

Remark 3.1. Hamiltonian operators of the shape (3.9) were classified by Dubrovin and Novikov [2] (see also [1,4]). In this case, the higher coefficient $a^{i j}$ must be a flat metric on manifold $M$ and $b_{k}^{i j}(u)$ are generated by this flat metric. These Hamiltonian operators play the very important role in the theory of integrability of the diagonalizable systems of hydrodynamic type $[5,6]$ (see also $[1,4]$ ).

Thus, any nonlinear sigma model (3.1) has a natural Hamiltonian (symplectic) representation (3.2) given by a symplectic operator (3.3). If a nonlinear sigma model system has also another Hamiltonian representation compatible with (3.2) then this nonlinear sigma model system will be integrable by the Lenart-Magri scheme ([37]). Thus, the problem of finding of Hamiltonian operators compatible with the symplectic operator (3.3) is very important and was formulated by author in 1989 (see $[8,11,38-40]$ ). For some known twocomponent integrable nonlinear sigma models without torsion $\left(r_{i j}\right.$ is a symmetric tensor in (3.1)) such second Hamiltonian representations compatible with (3.2), (3.3) were found in [41].

Example 3.1 [41].

$$
S=\int\left(\frac{1}{2} \frac{u_{x}^{1} u_{t}^{2}+u_{x}^{2} u_{t}^{1}}{u^{1} u^{2}+c}+k u^{1} u^{2}\right) d x d t
$$

where $c$ and $k$ are arbitrary constants.

In this case

$$
\begin{gathered}
\left(r_{i j}\right)=\frac{1}{u^{1} u^{2}+c}\left(\begin{array}{ll}
0 & 1 \\
1 & 0
\end{array}\right), \\
U(u)=k u^{1} u^{2}
\end{gathered}
$$

First symplectic operator (3.3) has the form

$$
A=\frac{1}{u^{1} u^{2}+c}\left(\begin{array}{cc}
0 & D \\
D & 0
\end{array}\right)-\frac{1}{\left(u^{1} u^{2}+c\right)^{2}}\left(\begin{array}{cc}
0 & u^{1} u_{x}^{2} \\
u^{2} u_{x}^{1} & 0
\end{array}\right)
$$

The second Hamiltonian operator $B$ for the action (3.14) is nonlocal and compatible with (3.15):

$$
\begin{gathered}
B=\left(\begin{array}{cc}
0 & -\left(u^{1} u^{2}+c\right) \\
\left(u^{1} u^{2}+c\right) & 0
\end{array}\right)+ \\
\left(\begin{array}{ccc}
u_{x}^{1} D^{-1} \circ u^{1} & -u_{x}^{1} D^{-1} \circ u^{2} \\
u_{x}^{2} D^{-1} \circ u^{1} & -u_{x}^{2} D^{-1} \circ u^{2}
\end{array}\right)+\left(\begin{array}{cc}
u^{1} D^{-1} \circ u_{x}^{1} & u^{1} D^{-1} \circ u_{x}^{2} \\
-u^{2} D^{-1} \circ u_{x}^{1} & -u^{2} D^{-1} \circ u_{x}^{2}
\end{array}\right)
\end{gathered}
$$


The recursion operator $L_{j}^{i}=B^{i s} A_{s j}$ for this integrable nonlinear sigma model (3.14) has the following form

$$
\begin{gathered}
L=\left(\begin{array}{cc}
-D & 0 \\
0 & D
\end{array}\right)+\frac{1}{u^{1} u^{2}+c}\left(\begin{array}{cc}
u^{1} u_{x}^{2} & 2 u^{1} u_{x}^{1} \\
-2 u^{2} u_{x}^{2} & -u^{2} u_{x}^{1}
\end{array}\right)+ \\
\left(\begin{array}{cc}
u_{x}^{1} D^{-1} \circ \frac{c u_{x}^{2}}{\left(u^{1} u^{2}+c\right)^{2}} & u_{x}^{1} D^{-1} \circ \frac{-c u_{x}^{1}}{\left(u^{1} u^{2}+c\right)^{2}} \\
u_{x}^{2} D^{-1} \circ \frac{c u_{x}^{2}}{\left(u^{1} u^{2}+c\right)^{2}} & u_{x}^{2} D^{-1} \circ \frac{-c u_{x}^{1}}{\left(u^{1} u^{2}+c\right)^{2}}
\end{array}\right)+ \\
\left(\begin{array}{cc}
u^{1} D^{-1} \circ\left(\frac{u^{1}\left(u_{x}^{2}\right)^{2}}{\left(u^{1} u^{2}+c\right)^{2}}-\frac{u_{x x}^{2}}{u^{1} u^{2}+c}\right) & u^{1} D^{-1} \circ\left(\frac{u^{2}\left(u_{x}^{1}\right)^{2}}{\left(u^{1} u^{2}+c\right)^{2}}-\frac{u_{x x}^{1}}{u^{1} u^{2}+c}\right) \\
-u^{2} D^{-1} \circ\left(\frac{u^{1}\left(u_{x}^{2}\right)^{2}}{\left(u^{1} u^{2}+c\right)^{2}}-\frac{u_{x x}^{2}}{u^{1} u^{2}+c}\right) & -u^{2} D^{-1} \circ\left(\frac{u^{2}\left(u_{x}^{1}\right)^{2}}{\left(u^{1} u^{2}+c\right)^{2}}-\frac{u_{x x}^{1}}{u^{1} u^{2}+c}\right)
\end{array}\right)
\end{gathered}
$$

Example 3.2 [41].

$$
S=\int\left(\frac{1}{2} \frac{u_{x}^{1} u_{t}^{2}+u_{x}^{2} u_{t}^{1}}{u^{1}+u^{2}}+k\left(u^{1}+u^{2}\right)\right) d x d t
$$

where $k$ is an arbitrary constant.

For this case

$$
\begin{gathered}
\left(r_{i j}\right)=\frac{1}{u^{1}+u^{2}}\left(\begin{array}{ll}
0 & 1 \\
1 & 0
\end{array}\right), \\
U(u)=k\left(u^{1}+u^{2}\right)
\end{gathered}
$$

The first symplectic operator (3.3) has the following form in this case:

$$
A=\frac{1}{u^{1}+u^{2}}\left(\begin{array}{cc}
0 & D \\
D & 0
\end{array}\right)-\frac{1}{\left(u^{1}+u^{2}\right)^{2}}\left(\begin{array}{cc}
0 & u_{x}^{2} \\
u_{x}^{1} & 0
\end{array}\right)
$$

The second Hamiltonian operator $B$ for the action (3.18) is also nonlocal and compatible with (3.19):

$$
\begin{gathered}
B=\left(\begin{array}{cc}
0 & -\left(u^{1}+u^{2}\right) \\
\left(u^{1}+u^{2}\right) & 0
\end{array}\right)+ \\
\left(\begin{array}{cc}
u_{x}^{1} D^{-1} & -u_{x}^{1} D^{-1} \\
u_{x}^{2} D^{-1} & -u_{x}^{2} D^{-1}
\end{array}\right)+\left(\begin{array}{cc}
D^{-1} \circ u_{x}^{1} & D^{-1} \circ u_{x}^{2} \\
-D^{-1} \circ u_{x}^{1} & -D^{-1} \circ u_{x}^{2}
\end{array}\right)
\end{gathered}
$$

The recursion operator $L_{j}^{i}=B^{i s} A_{s j}$ has the form

$$
\begin{gathered}
L=\left(\begin{array}{cc}
-D & 0 \\
0 & D
\end{array}\right)+\frac{1}{u^{1}+u^{2}}\left(\begin{array}{cc}
u_{x}^{2} & 2 u_{x}^{1} \\
-2 u_{x}^{2} & -u_{x}^{1}
\end{array}\right)+ \\
\left(\begin{array}{cc}
-u_{x}^{1} D^{-1} \circ \frac{u_{x}^{2}}{\left(u^{1}+u^{2}\right)^{2}} & u_{x}^{1} D^{-1} \circ \frac{u_{x}^{1}}{\left(u^{1}+u^{2}\right)^{2}} \\
-u_{x}^{2} D^{-1} \circ \frac{u_{x}^{2}}{\left(u^{1}+u^{2}\right)^{2}} & u_{x}^{2} D^{-1} \circ \frac{u_{x}^{1}}{\left(u^{1}+u^{2}\right)^{2}}
\end{array}\right)+ \\
\left(\begin{array}{cc}
D^{-1} \circ\left(\frac{\left(u_{x}^{2}\right)^{2}}{\left(u^{1}+u^{2}\right)^{2}}-\frac{u_{x x}^{2}}{u^{1}+u^{2}}\right) & D^{-1} \circ\left(\frac{\left(u_{x}^{1}\right)^{2}}{\left(u^{1}+u^{2}\right)^{2}}-\frac{u_{x x}^{1}}{u^{1}+u^{2}}\right) \\
D^{-1} \circ\left(-\frac{\left(u_{x}^{2}\right)^{2}}{\left(u^{1}+u^{2}\right)^{2}}+\frac{u_{x x}^{2}}{u^{1}+u^{2}}\right) & D^{-1} \circ\left(-\frac{\left(u_{x}^{1}\right)^{2}}{\left(u^{1}+u^{2}\right)^{2}}+\frac{u_{x x}^{1}}{u^{1}+u^{2}}\right)
\end{array}\right)
\end{gathered}
$$




\section{Homogeneous symplectic forms on loop spaces of manifolds}

Consider the matrix entries of the operator $A^{[m]}$ of the shape

$$
A_{i j}^{[m]}=a_{i j}^{[m]}(u) \frac{d^{m}}{d x^{m}}+b_{i j k}^{[m]}(u) u_{x}^{k} \frac{d^{m-1}}{d x^{m-1}}+\left(c_{i j k l}^{[m]}(u) u_{x}^{k} u_{x}^{l}+d_{i j k}^{[m]}(u) u_{x x}^{k}\right) \frac{d^{m-2}}{d x^{m-2}}+\ldots
$$

with all the terms in (4.1) being degree $m$ homogeneous with respect to the natural grading

$$
\begin{gathered}
\operatorname{deg}(f g)=\operatorname{deg} f+\operatorname{deg} g, \\
\operatorname{deg} f(u(x))=\operatorname{deg} u(x)=0, \\
\operatorname{deg} \frac{d^{k} u}{d x^{k}}=\operatorname{deg} \frac{d^{k}}{d x^{k}}=k
\end{gathered}
$$

Symplectic operators of the shape (4.1) and the corresponding symplectic forms on the loop space $L(M)$

$$
\omega(\xi, \eta)=\int_{S^{1}} \xi^{i}\left(A_{i j}^{[m]} \eta^{j}\right) d x
$$

were introduced and studied by author in $[9,38,39]$.

It can be proved easily that for symplectic form (4.2) the coefficients of the operator (4.1) are transformed under changes of coordinates $u^{i}=u^{i}(v)$ on the $N$-dimensional manifold $M$ as differential geometric objects. Under the assumption of nondegeneracy of the higher coefficient $a_{i j}^{[m]}(u)$ the conditions reflecting symplecticity of the operator (4.1) are in their essence some natural differential-geometric restrictions imposed on connections defined on manifold $M$ equipped with the metric $a_{i j}^{[m]}$ (symmetric for any odd $m, m=2 k+1$, or skewsymmetric for any even $m, m=2 k$ ) and the coefficients can be expressed in an invariant form via the metric, the curvatures and the torsions of the connections. It allows to obtain invariant geometric description of the symplectic operators (4.1).

Definition 4.1. Symplectic operators of the shape (4.1) are called symplectic operators of differential geometric type.

Remark 4.1. Poisson structures of the shape (4.1) were introduced by Dubrovin and Novikov [3] as a natural generalization of Poisson structures of hydrodynamic type $(m=1)$. The case $m=2$ for Poisson structures of the shape (4.1) was studied in [44-46].

For $m=0$ symplecticity conditions are

$$
\begin{gathered}
a_{i j}^{[0]}(u)=-a_{j i}^{[0]}(u), \\
\sum_{(i, j, k)} \frac{\partial a_{i j}^{[0]}}{\partial u^{k}}=0,
\end{gathered}
$$

where the sum is taken with respect to all cyclic permutations of $i, j, k$. It means that

$$
\Omega_{0}=a_{i j}^{[0]}(u) d u^{i} \wedge d u^{j}
$$


is an arbitrary closed 2 -form on $M$. If $\operatorname{det} a_{i j}^{[0]} \neq 0$, we have a symplectic structure $\Omega_{0}$ on $M$ and a symplectic structure

$$
\omega(\xi, \eta)=\int_{S^{1}} \Omega_{0}(\xi, \eta) d x
$$

on the loop space $L(M)$.

The case $m=1$ corresponds exactly to symplectic forms (3.4). It is possible also to get a complete description of matrix symplectic operators (4.1) for $m=2$ [9]. In this case we deal with the operators of the form

$$
A_{i j}^{[2]}=a_{i j}^{[2]}(u) \frac{d^{2}}{d x^{2}}+b_{i j k}^{[2]}(u) u_{x}^{k} \frac{d}{d x}+c_{i j k l}^{[2]}(u) u_{x}^{k} u_{x}^{l}+d_{i j k}^{[2]}(u) u_{x x}^{k}
$$

Lemma 4.1. Operator (4.3) is symplectic if and only if the following conditions are valid:

$$
\begin{gathered}
a_{i j}^{[2]}=-a_{j i}^{[2]}, \\
\frac{\partial a_{i j}^{[2]}}{\partial u^{k}}=\frac{1}{2}\left(b_{i j k}^{[2]}-b_{j i k}^{[2]}\right), \\
d_{i j k}^{[2]}=\frac{1}{2}\left(b_{i j k}^{[2]}+b_{k j i}^{[2]}-b_{k i j}^{[2]}\right), \\
c_{i j k l}^{[2]}+c_{i j l k}^{[2]}=\frac{\partial d_{i j k}^{[2]}}{\partial u^{l}}+\frac{\partial d_{i j l}^{[2]}}{\partial u^{k}}+\frac{\partial d_{l i k}^{[2]}}{\partial u^{j}}-\frac{\partial d_{l j k}^{[2]}}{\partial u^{i}} .
\end{gathered}
$$

Theorem $4.1[9]$. Under the assumption $\operatorname{det} a_{i j}^{[2]}(u) \neq 0$, symplectic operators of the form (4.3) exist if and only if $M$ is an even-dimensional manifold with an almost symplectic structure.

We remind that a manifold with an almost symplectic structure is an even-dimensional $M$ endowed with a nondegenerate skew-symmetric metric $g_{i j}(u)$.

Let $a_{i j}^{[2]}(u)=g_{i j}(u)$. Consider an arbitrary symplectic operator of the form (4.2) and introduce coefficients $\Gamma_{j k}^{i}(u)$ by the relation

$$
b_{i j k}^{[2]}(u)=2 g_{i s}(u) \Gamma_{j k}^{s}(u) .
$$

Lemma 4.2 [9]. Coefficients $\Gamma_{j k}^{i}(u)$ define a symplectic connection on $\left(M, g_{i j}\right)$, i.e. a differential geometric connection compatible with the almost symplectic structure of the manifold:

$$
\nabla_{k} g_{i j} \equiv \frac{\partial g_{i j}}{\partial u^{k}}-g_{i s} \Gamma_{j k}^{s}-g_{j s} \Gamma_{i k}^{s}=0
$$

It turns out that any symplectic connection $\Gamma_{j k}^{i}(u)$ on a manifold with an almost symplectic structure $\left(M, g_{i j}\right)$ generates a unique symplectic operator (4.3).

Theorem 4.2 [9]. If $\left(M, g_{i j}\right)$ is a manifold with an almost symplectic structure then there exists a one-to-one correspondence between the symplectic connections $\Gamma_{j k}^{i}$ on $\left(M, g_{i j}\right)$ and closed 2 -forms of the shape $(4.2), m=2$, on the space $L(M)$ of smooth loops on $M$. This correspondence is expressed by the formula

$$
\omega(\xi, \eta)=\int_{S^{1}}\left\{<\xi, \nabla_{\nu}^{2} \eta>+\frac{1}{2}<\nu, R(\xi, \eta) \nu>+<\nu, \sum_{(\eta, \xi, \nu)} R(\eta, \xi) \nu>\right.
$$




$$
\left.+<T(\nu, \xi), T(\nu, \eta)>+<T(\xi, \eta), \nabla_{\nu} \nu>\right\} d x
$$

where $\left\langle\xi, \eta>=g_{i j} \xi^{i} \eta^{j}\right.$, the sum $\sum_{(\eta, \xi, \nu)}$ is taken with respect to all cyclic permutations of the elements $(\eta, \xi, \nu), \quad \nu$ is the velocity vector of the loop $\gamma(x)$, i.e. $\nu^{i}=u_{x}^{i}, \nabla_{\nu}$ is the covariant derivation along $\gamma,[T(\xi, \eta)]^{i}=T_{k l}^{i} \xi^{k} \eta^{l}, T_{j k}^{i} \equiv \Gamma_{j k}^{i}-\Gamma_{k j}^{i}$, is the torsion tensor of the connection and $[R(\xi, \eta) \zeta]^{i}=R_{j k l}^{i} \xi^{k} \eta^{l} \zeta^{j}$,

$$
R_{j k l}^{i}=-\frac{\partial \Gamma_{j l}^{i}}{\partial u^{k}}+\frac{\partial \Gamma_{j k}^{i}}{\partial u^{l}}-\Gamma_{p k}^{i} \Gamma_{j l}^{p}+\Gamma_{p l}^{i} \Gamma_{j k}^{p},
$$

is the curvature tensor of the connection.

The formula (4.8) gives the general form of closed 2-forms of the shape (4.2), $\mathrm{m}=2$, and also describes all the symplectic operators of the form (4.3).

On symplectic manifolds there is a special class of symmetric symplectic connections, satisfying the condition $T_{j k}^{i}=0$. Such connections exist on $\left(M, g_{i j}\right)$ if and only if the manifold $\left(M, g_{i j}\right)$ is in fact a symplectic one, i.e., $(d g)_{i j k}=0$.

Corollary 4.1 [9]. If a symplectic connection $\Gamma_{j k}^{i}(u)$ is a symmetric one then the closed 2 -form (4.8) has the presentation

$$
\omega(\xi, \eta)=\int_{S^{1}}\left\{<\xi, \nabla_{\nu}^{2} \eta>+\frac{1}{2}<\nu, R(\xi, \eta) \nu>\right\} d x
$$

This formula describes a most natural class of homogeneous symplectic forms of the second order on loop spaces of symplectic manifolds.

It is worth being noted that the class of symplectic connections on manifolds with almost symplectic structures is rich enough. Namely, any such a manifold has infinitely many distinct symplectic connections and correspondingly infinitely many distinct symplectic forms (4.8) on its loop space. Similarly, any symplectic manifold has infinitely many symmetric symplectic connections and correspondingly symplectic forms of the shape (4.9) on its loop space.

\section{Lagrangian systems and symplectic structures on loop spaces}

We shall consider in this section two-dimensional Lagrangian systems generated by the actions of the form $([14,12])$

$$
S=\int\left(g_{i}\left(x, u, u_{x}, \ldots\right) u_{t}^{i}+h\left(x, u, u_{x}, \ldots\right)\right) d x d t
$$

where $g_{i}, h$ are arbitrary functions depending on the independent space variable $x$, some $N$ fields $u^{i}(x, t)$ and their derivatives $u_{(n)}^{i}=\partial^{n} u^{i} / \partial x^{n}$ with respect to $x$.

The corresponding Lagrangian system

$$
\frac{\delta S}{\delta u^{i}}=0
$$

has the following form

$$
M_{i j} u_{t}^{j}=\frac{\delta H}{\delta u^{i}}
$$


where

$$
M_{i j}=\frac{\partial g_{i}}{\partial u_{(n)}^{j}} \frac{d^{n}}{d x^{n}}-(-1)^{n} \frac{d^{n}}{d x^{n}} \circ \frac{\partial g_{j}}{\partial u_{(n)}^{i}}
$$

and

$$
H=\int h d x
$$

Lemma 5.1 $[\mathbf{1 2 , 1 4}]$. The differential operator $M_{i j}$ is a symplectic operator and the bilinear form

$$
\omega(\xi, \eta)=\int_{S^{1}} \xi^{i} M_{i j} \eta^{j} d x
$$

where $\xi, \eta \in T_{\gamma} L(M)$, is a symplectic form on the loop space $L(M)$.

In other words, the inverse operator $K^{i j}=\left(M^{-1}\right)^{i j}$, such that $K^{i j} M_{j k}=\delta_{k}^{i}$, gives always a nonlocal Poisson bracket

$$
\left\{u^{i}(x), u^{j}(y)\right\}=K^{i j}[u(x)] \delta(x-y)
$$

and hence we have always the following nonlocal Hamiltonian representation for Lagrangian systems generated by the actions (5.1):

$$
u_{t}^{i}=K^{i j} \frac{\delta H}{\delta u^{j}} \equiv\left\{u^{i}(x), H\right\}
$$

The corresponding symplectic representation for these Lagrangian systems has the form $([14])$

$$
\omega\left(\delta u, u_{t}\right)=\delta H
$$

where the relation (5.7) is valid for arbitrary variations $\delta u^{i}(x)$ of the fields $u^{i}(x), H$ is a functional on the loop space $L(M)$.

Some special actions of the form (5.1) generate important nonlinear equations of mathematical physics and the field theory such as nonlinear sigma models (see above section 3 ), Monge-Ampère equations, some systems of hydrodynamic type, Krichever-Novikov equation and so on and the corresponding symplectic representations are very useful and effective for investigation of integrability and the procedures of averaging. Of course, it is easy to generalize this construction for arbitrary degenerate Lagrangian quasilinear with respect to higher $t$-derivatives of the fields $u^{i}(x, t)$.

Let's consider some examples.

Example 5.1. The Korteweg-de Vries equation (KdV).

Consider the following simple action of the type (5.1)

$$
S=\int\left(\frac{1}{2} u_{x} u_{t}-\frac{1}{2} u_{x x}^{2}-u_{x}^{3}\right) d x d t
$$

The symplectic representation (5.2)-(5.3) for the corresponding Lagrangian equation $\delta S / \delta u=0$ has the form

$$
\begin{gathered}
\frac{d}{d x}\left(u_{t}\right)=-\frac{\delta H}{\delta u}, \\
H=\int\left(u_{x}^{3}+\frac{1}{2} u_{x x}^{2}\right) d x
\end{gathered}
$$


After the field transformation $v(x)=u_{x}(x)$ we have obtained the usual Hamiltonian representation of the $\mathrm{KdV}$ equation:

$$
\begin{gathered}
v_{t}=\frac{d}{d x} \frac{\delta H}{\delta v} \\
H=\int\left(v^{3}+\frac{1}{2} v_{x}^{2}\right) d x \\
v_{t}=6 v v_{x}-v_{x x x}
\end{gathered}
$$

Remark 5.1. Note that if $v^{i}(x)=u_{x}^{i}(x)$ then for any functional $H$ the relation

$$
\frac{\delta H}{\delta u^{i}}=-\frac{d}{d x} \frac{\delta H}{\delta v^{i}}
$$

is valid for the variation derivatives.

Example 5.2 [10]. The Krichever-Novikov equation (KN).

Consider the special action of the form (5.1)

$$
S=\int\left(\frac{1}{2} \frac{u_{t}}{u_{x}}-\frac{1}{2} \frac{u_{x x}^{2}}{u_{x}^{2}}-\frac{1}{3} \frac{R(u)}{u_{x}^{2}}\right) d x d t
$$

where $R(u)=a_{3} u^{3}+a_{2} u^{2}+a_{1} u+a_{0}$ is an arbitrary polynomial of degree three, $a_{i}=$ const, $i=0,1,2,3$.

In this case the Lagrangian equation $\delta S / \delta u=0$ has the symplectic representation (5.2)-(5.3) with the symplectic operator

$$
\begin{gathered}
M=-\frac{1}{u_{x}} \frac{d}{d x} \circ \frac{1}{u_{x}} \\
\left(\frac{1}{u_{x}} \frac{d}{d x} \frac{1}{u_{x}}\right) u_{t}=\frac{\delta H}{\delta u}, \\
H=\int\left(\frac{1}{2} \frac{u_{x x}^{2}}{u_{x}^{2}}+\frac{1}{3} \frac{R(u)}{u_{x}^{2}}\right) d x
\end{gathered}
$$

The formulas (5.10)-(5.11) give the well-known symplectic (and Hamiltonian) representation ([20]) for the Krichever-Novikov equation ([21])

$$
u_{t}=u_{x x x}-\frac{3}{2} \frac{u_{x x}^{2}}{u_{x}}+\frac{R(u)}{u_{x}},
$$

which is a completely integrable equation with an infinite collection of conservation laws, a representation of zero curvature with parameter on an elliptic curve and so on, and plays an important role in the contemporary theory of integrable nonlinear systems of mathematical physics.

We recall that the equation (5.12) appeared for the first time in [21] (in slightly other form, see also $[22,23]$ ) as the deformation equation (by virtue of the Kadomtsev-Petviashvili (KP) equation) for the Tyurin parameters corresponding to a general solution of the commutation equation of ordinary differential operators for a spectral curve of genus $g=1$ and 
fixed rank $l=2$ of the vector bundle of common eigenfunctions over the spectral curve (we note that the corresponding integrable Boussinesq type system of deformation of Tyurin parameters by virtue of KP equation for general solution of the commutation equation of ordinary differential operators of genus $g=1$ and rank $l=3$ is obtained in author's works $[24,25,13])$. Correspondingly any solution of the KN equation determines a solution of $\mathrm{KP}$ by an explicit formula $[21,22]$. It is shown in $[26,27]$ that among all nonlinear equations (up to local changes of the field variable $u=u(v)$ ) of the Korteweg-de Vries type:

$$
u_{t}=u_{x x x}+f\left(u, u_{x}, u_{x x}\right) \text {, }
$$

having infinite series of local conservation laws, only the KN equation (in general case, i.e., when the polynomial $R(u)$ has no multiple roots) can not be reduced to the KdV equation by differential substitutions of the form

$$
u=F\left(v, v_{x}, \ldots, v_{(m)}\right)
$$

although, generally speaking, the question of possible connection between the $\mathrm{KN}$ and $\mathrm{KdV}$ equations by more complicated transformations is still unsolved.

The KN equation appeared as the first nonformal example of a system whose Hamiltonian structure is defined by a nontrivial differential symplectic operator. The action (5.8) gives Lagrangian representation for the KN equation ([10]) and explains natural origin of the symplectic operator (5.9).

The KN equation (5.12) is related with the Hamiltonian equation ([10])

$$
\begin{gathered}
v_{t}=-\left(\frac{d}{d z}\right)^{-1} \frac{\delta H}{\delta v} \\
H=-\int\left[\frac{1}{2} \frac{v_{z z}^{2}}{v_{z}^{3}}+\frac{1}{3} R(z) v_{z}^{3}\right] d z
\end{gathered}
$$

by the point transformation of hodograph type

$$
\left\{\begin{array}{l}
x=v \\
u=z
\end{array}\right.
$$

One can assume that $v(z)$ is a new scalar field which is the inverse of the field $u(x)$, i.e., $u(v(z))=z$ and $v(u(x))=x$.

Introducing the new field $w(z)=v_{z}(z)$ we obtain a canonical Hamiltonian representation of the Krichever-Novikov equation (5.12) ([10]):

$$
\begin{gathered}
w_{t}=\frac{d}{d z} \frac{\delta H}{\delta w} \\
H=-\int\left[\frac{1}{2} \frac{w_{z}^{2}}{w^{3}}+\frac{1}{3} R(z) w^{3}\right] d z .
\end{gathered}
$$

Note that the Hamiltonian $H$ is not invariant with respect to translations in $z$.

As it was shown by author in [10] the KN equation is simply connected also with the canonical Hamiltonian system

$$
\left\{\begin{array}{l}
q_{t}=\frac{\delta F}{\delta p} \\
p_{t}=-\frac{\delta F}{\delta q}
\end{array}\right.
$$


where the Hamiltonian $F[p, q]$ in conjugate variables $p(z), q(z)$ has the form

$$
\begin{gathered}
F[p, q]= \\
\int\left(p(z)\left(\frac{q_{z z z}}{q_{z}^{3}}-\frac{3}{2} \frac{q_{z z}^{2}}{q_{z}^{4}}-R(z) q_{z}^{2}\right)+\frac{1}{3} R(z) q_{z}^{3}-\frac{3}{2} \frac{q_{z z}^{2}}{q_{z}^{3}}\right) d z
\end{gathered}
$$

In $[28,29,30]$ symplectic structures of the degenerate $\mathrm{KN}$ equation $(R(u) \equiv 0)$ are considered (as it is known ([27]), in this case and always, when the polynomial $R(u)$ has multiple roots, the $\mathrm{KN}$ equation reduces to the $\mathrm{KdV}$ equation by differential substitution). The degenerate KN equation has the form

$$
u_{t}=u_{x} S[u]
$$

where

$$
S[u]=\frac{u_{x x x}}{u_{x}}-\frac{3}{2} \frac{u_{x x}^{2}}{u_{x}^{2}}
$$

is the Schwarzian of $u$, and has a compatible pair of differential symplectic operators found by Dorfman $([28-30,12])$ and a number of other interesting properties studied by various authors (see [31], for example). For multicomponent analog of the degenerate KN equation (5.20) multisymplectic representation is found by Fordy and Antonowicz [32]. In [63] a $(2+1)$-dimensional version of the degenerate $\mathrm{KN}$ equation (5.20) was studied by Dorfman and Nijhoff.

It is interesting that as it was discovered by author and Ferapontov ([15], see also [16-17] and sections 7 and 9 in this paper) the symplectic operator (5.9) and its multicomponent generalization

$$
M_{i j}=\frac{1}{K} \frac{1}{u_{x}^{i}} \frac{d}{d x} \circ \frac{1}{u_{x}^{j}},
$$

$K=$ const, arises naturally in the Dubrovin-Novikov theory of Hamiltonian systems of hydrodynamic type developed in [1-4]. The Hamiltonian structure of these systems introduced and studied by Dubrovin and Novikov in [2] is generated by metrics of zero curvature (all these Hamiltonian systems of hydrodynamic type have a natural Lagrangian representation of the considered above type (5.1)-(5.3) and we shall consider them in the section 7). It was shown in [15] that with the help of the operator (5.21) one can adequately extend the theory of Hamiltonian systems of hydrodynamic type to metrics of arbitrary constant Riemannian curvature $K$. In particular, such Hamiltonian diagonalizable systems of hydrodynamic type will be integrable (they are semi-Hamiltonian in the sense of Tsarev [5-6]). All systems of hydrodynamic type derived by averaging of well-known integrable equations of the soliton theory (KdV, Nonlinear Schrödinger, Sine-Gordon and so on) have Hamiltonian structures generated by metrics of non-zero constant Riemanian curvature (along with structures of Dubrovin-Novikov type) (see [19]).

\section{Symplectic and Poisson structures of the Monge-Ampère equations}

Example 6.1. The Monge-Ampère equations. 
Consider the following action of type (5.1) [50]

$$
S=\int\left[\frac{1}{2} u_{x}^{2} q_{t}-u_{x} q_{x} u_{t}-\frac{1}{2} q^{2} u_{x x}+\Phi\left(x, t, u, u_{x}\right)\right] d x d t
$$

where $\Phi\left(x, t, u, u_{x}\right)$ is an arbitrary function.

The corresponding Lagrangian system $\delta S / \delta u^{i}=0$ has natural symplectic representation (5.2)-(5.3) of the form

$$
M\left(\begin{array}{l}
u \\
q
\end{array}\right)_{t}=\left(\begin{array}{l}
\delta F / \delta u \\
\delta F / \delta q
\end{array}\right)
$$

where

$$
M=\left(\begin{array}{cc}
q_{x} \frac{d}{d x}+\frac{d}{d x} \circ q_{x} & -u_{x x} \\
u_{x x} & 0
\end{array}\right)
$$

is a symplectic operator,

$$
F=\int\left[\frac{1}{2} q^{2} u_{x x}-\Phi\left(x, t, u, u_{x}\right)\right] d x
$$

The symplectic system (6.2)-(6.4) is equivalent to the Monge-Ampère equation ([50])

$$
u_{x x} u_{t t}-\left(u_{x t}\right)^{2}=\frac{\delta \Phi}{\delta u}
$$

In this case of the Monge-Ampère equations the Poisson bracket (5.5) is also local and corresponding Hamiltonian operator $K^{i j}=\left(M^{-1}\right)^{i j}$ has the form $([50])$

$$
K=\left(\begin{array}{cc}
0 & \frac{1}{u_{x x}} \\
-\frac{1}{u_{x x}} & \frac{q_{x}}{u_{x x}^{2}} \frac{d}{d x}+\frac{d}{d x} \circ \frac{q_{x}}{u_{x x}^{2}}
\end{array}\right)
$$

And what is more this fact is valid for all local Hamiltonian operators of the form

$$
K=\left(\begin{array}{cc}
0 & B \\
-B & A \frac{d}{d x}+A \circ \frac{d}{d x}
\end{array}\right)
$$

where $B$ and $A$ are some functions of $x, u, u_{x}, u_{x x}, \ldots, q, q_{x}, q_{x x}, \ldots \quad(B \neq 0)$, which were considered by Nutku and Sarıoğlu in [50] in connection with Poisson structures of the Monge-Ampère equations. Namely, for any local Hamiltonian differential operator of the form (6.7) the corresponding inverse symplectic operator $M$ is also local differential operator and has the form

$$
M=\left(\begin{array}{cc}
\frac{A}{B^{2}} \frac{d}{d x}+\frac{d}{d x} \circ \frac{A}{B^{2}} & -\frac{1}{B} \\
\frac{1}{B} & 0
\end{array}\right)
$$

Theorem 6.1. The operator

$$
M=\left(\begin{array}{cc}
{\left[\frac{\partial g}{\partial u_{x}}-\left(\frac{\partial g}{\partial u_{x x}}\right)_{x}\right] \frac{d}{d x}+\frac{d}{d x} \circ\left[\frac{\partial g}{\partial u_{x}}-\left(\frac{\partial g}{\partial u_{x x}}\right)_{x}\right]} & \frac{\partial g}{\partial q} \\
-\frac{\partial g}{\partial q} & 0
\end{array}\right)
$$


is a symplectic operator of the form (6.8) for arbitrary function $g\left(x, t, u, u_{x}, u_{x x}, q\right)$. The corresponding local Hamiltonian operator $K$ has the form (6.7) where

$$
B=-\frac{1}{(\partial g / \partial q)}, \quad A=\frac{\frac{\partial g}{\partial u_{x}}-\left(\frac{\partial g}{\partial u_{x x}}\right)_{x}}{(\partial g / \partial q)^{2}}
$$

\section{Symplectic and nonlocal Poisson structures of homogeneous and nonhomoge- neous systems of hydrodynamic type}

Example 7.1. Systems of hydrodynamic type.

Consider the following action of type (5.1)

$$
S=\int\left(\frac{1}{2} g_{i j} u_{x}^{i} u_{t}^{j}-h\left(u_{x}\right)\right) d x d t
$$

where $\left(g_{i j}\right)$ is a constant nondegenerate symmetric tensor, $h(v)$ is an arbitrary function.

The corresponding Lagrangian system $\delta S / \delta u^{i}=0$ has the symplectic representation (5.2)-(5.3) of the form

$$
\begin{gathered}
g_{i j} \frac{d}{d x}\left(u_{t}^{j}\right)=-\frac{\delta H}{\delta u^{i}}, \\
H=\int h\left(u_{x}\right) d x
\end{gathered}
$$

After the field transformation $v^{i}(x)=u_{x}^{i}(x)$ (see Remark 5.1) we obtain a Hamiltonian system of hydrodynamic type in flat coordinates

$$
\begin{gathered}
v_{t}^{i}=g^{i j} \frac{d}{d x} \frac{\delta H}{\delta v^{j}}, \\
H=\int h(v) d x
\end{gathered}
$$

In other words, the action (7.1) gives the general class of Hamiltonian systems of hydrodynamic type (written in flat coordinates $\left(v^{1}, \ldots, v^{N}\right)$ ), introduced and studied by Dubrovin and Novikov [1-4].

We remind very briefly the basic necessary for us notions and results on Poisson structures of hydrodynamic type.

Consider the one-dimensional systems of hydrodynamic type, i.e., in other words, the one-dimensional evolution quasilinear systems of the first order

$$
u_{t}^{i}=v_{j}^{i}(u) u_{x}^{j},
$$

where $v_{j}^{i}(u)$ is an arbitrary $N \times N$ matrix function of $u=\left(u^{1}, \ldots, u^{N}\right), u^{i}=u^{i}(x, t), i=$ $1, \ldots, N$.

The Hamiltonian systems of hydrodynamic type considered by Dubrovin and Novikov in [1-4] have the form

$$
u_{t}^{i}=\left\{u^{i}, H\right\},
$$


where $H$ is a functional of hydrodynamic type, i.e.

$$
H=\int h(u) d x
$$

and the Poisson bracket has the form

$$
\left\{u^{i}(x), u^{j}(y)\right\}=g^{i j}(u(x)) \delta_{x}(x-y)+b_{k}^{i j}(u(x)) u_{x}^{k} \delta(x-y)
$$

(the Poisson bracket of Dubrovin-Novikov type [2]). It was shown in [2] that if $\operatorname{det}\left[g^{i j}(u)\right] \neq 0$ then the expression (7.7) gives a Poisson bracket if and only if

(1) $g^{i j}(u)$ is a metric of zero Riemannian curvature (that is, simply a flat metric),

(2) $b_{k}^{i j}(u)=-g^{i s}(u) \Gamma_{s k}^{j}(u)$, where $\Gamma_{s k}^{j}(u)$ are the coefficients of the differential geometric connection generated by the metric $g^{i j}$, that is, the only symmetric connection compatible with the metric (the Levi-Civita connection).

Thus, there exist always local variables $v^{i}=v^{i}(u)$ in which the Poisson bracket (7.7) is simply constant:

$$
\left\{v^{i}(x), v^{j}(y)\right\}=\varepsilon^{i} \delta^{i j} \delta_{x}(x-y),
$$

where $\varepsilon^{i}= \pm 1, i=1, \ldots, N$. Correspondingly, it is easy to give a Lagrangian description (7.1) to these systems. The considered Hamiltonian systems of hydrodynamic type have the form

$$
u_{t}^{i}=\left[\nabla^{i} \nabla_{j} h(u)\right] u_{x}^{j}
$$

where $\nabla$ is the covariant derivative generated by a zero curvature metric. As it was shown in $[5,6]$, if the Hamiltonian system of hydrodynamic type (7.9) has the Riemann invariants (that is, the matrix $v_{j}^{i}(u)=\nabla^{i} \nabla_{j} h(u)$ is diagonalizable), then it is integrable.

Multidimensional Poisson structures of hydrodynamic type were introduced and studied in $[3,7]$.

In $[15]$ (see also $[16,17]$ ) there was proposed a nonlocal generalization of Hamiltonian theory of the systems of hydrodynamic type (7.4) connected with the nonlocal Poisson brackets of the form

$$
\begin{gathered}
\left\{u^{i}(x), u^{j}(y)\right\}= \\
g^{i j}(u(x)) \delta_{x}(x-y)+b_{k}^{i j}(u(x)) u_{x}^{k} \delta(x-y)+K u_{x}^{i}(d / d x)^{-1} u_{x}^{j} \delta(x-y) .
\end{gathered}
$$

It is simple to show that for any Hamiltonian functional $H$ of hydrodynamic type $\left(H=\int h(u) d x\right)$ the Poisson bracket of the form (7.10) also generates always a system of hydrodynamic type (7.4).

Remark 7.1 [16]. The expression (7.10) is the most general form of Poisson brackets with the property to generate a system of hydrodynamic type (7.4) for any Hamiltonian $H$ of hydrodynamic type (7.6).

Theorem 7.1 [15] If $\operatorname{det}\left[g^{i j}(u)\right] \neq 0$ then expression (7.10) gives a Poisson bracket if and only if

(1) $g^{i j}(u)$ is a metric of constant Riemannian curvature $K$,

(2) $b_{k}^{i j}(u)=-g^{i s} \Gamma_{s k}^{j}(u)$, where $\Gamma_{s k}^{j}(u)$ are the coefficients of the connection generated by the metric $g^{i j}$ (the Levi-Civita connection). 
The corresponding Hamiltonian systems of hydrodynamic type have the form

$$
u_{t}^{i}=\left[\nabla^{i} \nabla_{j} h(u)+K \delta_{j}^{i} h(u)\right] u_{x}^{j},
$$

where $\nabla$ is the covariant derivative generated by a metric of constant Riemannian curvature $K$.

If $\operatorname{det}\left[g^{i j}(u)\right]=0$ then description of the nonlocal Poisson brackets (7.10) much more complicated [16].

Note that the Poisson brackets (7.7) and (7.10) define natural differential geometric Poisson brackets on loop spaces of arbitrary flat and constant Riemannian curvature manifolds correspondingly by analogy with symplectic structures of differential geometric type on loop spaces of arbitrary Riemannian manifolds [8,9] (see also (3.4) and the sections 2-4 in this paper).

Consider now nonhomogeneous systems of hydrodynamic type

$$
u_{t}^{i}=v_{j}^{i}(u) u_{x}^{j}+f^{i}(u) .
$$

Local Poisson structures for the systems (7.12) studied by Dubrovin and Novikov [3,4] have also a natural nonlocal generalization. We shall consider here nonlocal Hamiltonian nonhomogeneous systems of hydrodynamic type

$$
u_{t}^{i}=\left\{u^{i}, H\right\},
$$

where $H$ is a functional of hydrodynamic type (7.6), and the nonlocal nonhomogeneous Poisson bracket of hydrodynamic type has the form

$$
\begin{gathered}
\left\{u^{i}(x), u^{j}(y)\right\}=g^{i j}(u(x)) \delta_{x}(x-y)+b_{k}^{i j}(u(x)) u_{x}^{k} \delta(x-y)+ \\
K u_{x}^{i}(d / d x)^{-1} u_{x}^{j} \delta(x-y)+\omega^{i j}(u(x)) \delta(x-y)
\end{gathered}
$$

It is easy to show that the expression (7.14) is a Poisson bracket if and only if it is a sum of two compatible Poisson brackets (7.10) and (1.3).

Theorem 7.2 [52]. The Poisson brackets (7.10) and (1.3) are compatible if and only if the bivector $\omega^{i j}(u)$ is a Killing bivector on the manifold $\left(M, g^{i j}\right)$ of constant Riemannian curvature $K$, that is,

$$
\nabla^{i} \omega^{j k}+\nabla^{j} \omega^{i k}=0,
$$

where $\nabla$ is the covariant derivative generated by the metric $g^{i j}(u)$ of constant Riemannian curvature $K$.

\section{On some integrable nonhomogeneous systems of hydrodynamic type}

In this section we shall consider some special class of nonhomogeneous systems of hydrodynamic type with quadratic nonlinearity $([49,48])$ :

$$
u_{t}^{i}=a^{i} u_{x}^{i}+\sum_{k, j} b_{j k}^{i} u^{j} u^{k}+\sum_{k} c_{k}^{i} u^{k},
$$


where a summation over repeating indices is not assumed in this section, $a^{i}, b_{j k}^{i}$ and $c_{k}^{i}$ are constant tensors, $i, j, k=1, \ldots, N$.

There are a number of well-known integrable systems among (8.1).

Example 8.1. The N-wave equation.

For example, integrable real-valued exact resonance system of parametric interaction of three wave packets in nonlinear optics $(N=3)$ is a special case of $(8.1)$ :

$$
\left\{\begin{array}{l}
u_{t}^{1}=a^{1} u_{x}^{1}-\varepsilon u^{2} u^{3} \\
u_{t}^{2}=a^{2} u_{x}^{2}+\varepsilon u^{1} u^{3} \\
u_{t}^{3}=a^{3} u_{x}^{3}+\varepsilon u^{1} u^{2}
\end{array}\right.
$$

where $a^{i}, \varepsilon$ are some constants.

Example 8.2. The KdV equation.

Consider the KdV equation (see also Example 5.1) as evolution system with respect to $x$ :

$$
\left\{\begin{array}{l}
u_{x}^{1}=u^{2} \\
u_{x}^{2}=u^{3} \\
u_{x}^{3}=-u_{t}^{1}+6 u^{1} u^{2}
\end{array}\right.
$$

It was shown in [47] that the KdV system (8.3) is Hamiltonian with respect to some nonhomogeneous Poisson structures of hydrodynamic type (7.14) which are in fact induced by well-known Magri and Gardner brackets for KdV. After the local quadratic unimodular change of field variables [48]

$$
\left\{\begin{array}{l}
u^{1}=\left(w^{1}-w^{3}\right) / \sqrt{2} \\
u^{2}=w^{2} \\
u^{3}=\left(w^{1}+w^{3}\right) / \sqrt{2}+\left(w^{1}-w^{3}\right)^{2}
\end{array}\right.
$$

we obtain Hamiltonian representation for the $\mathrm{KdV}$ system (8.3) ([48]), generated by the simplest infinite dimensional Kac-Moody Lie algebra $\widehat{s l}(2)$ and some quadratic Hamiltonian $H$ :

$$
\begin{gathered}
w_{x}^{i}=M^{i j}(t) \frac{\delta H}{\delta w^{j}}, \\
H=-\int\left[\left(w^{1}-w^{3}\right)^{2}-\sqrt{2}\left(w^{1}+w^{3}\right)\right] d t \\
\left(M^{i j}(t)\right)=\left(\begin{array}{ccc}
1 & 0 & 0 \\
0 & -1 & 0 \\
0 & 0 & -1
\end{array}\right) \frac{d}{d t}+\left(\begin{array}{ccc}
0 & -2 w^{3}(t) & 2 w^{2}(t) \\
2 w^{3}(t) & 0 & 2 w^{1}(t) \\
-2 w^{2}(t) & -2 w^{1}(t) & 0
\end{array}\right),
\end{gathered}
$$

where $M$ is the Hamiltonian operator given by $\widehat{s l}(2)$ [48].

The second nonhomogeneous Poisson structure of hydrodynamic type for the $\mathrm{KdV}$ system (8.3) is compatible with (8.6) and has the following form [48]:

$$
\left(L^{i j}(t)\right)=\frac{1}{2}\left(\begin{array}{lll}
1 & 0 & 1 \\
0 & 0 & 0 \\
1 & 0 & 1
\end{array}\right) \frac{d}{d t}+
$$




$$
\left(w^{1}-w^{3}\right)\left(\begin{array}{ccc}
0 & 1 & 0 \\
-1 & 0 & -1 \\
0 & 1 & 0
\end{array}\right)+\frac{1}{\sqrt{2}}\left(\begin{array}{ccc}
0 & 1 & 0 \\
-1 & 0 & 1 \\
0 & -1 & 0
\end{array}\right)
$$

here the metric $g^{i j}(w)$ in (8.7) is degenerate (see (7.14)).

Correspondingly, second Hamiltonian representation for the KdV system (8.3) has the form

$$
w_{x}^{i}=L^{i j}(t) \frac{\delta H}{\delta w^{j}}
$$

where $H$ is the quadratic Hamiltonian

$$
H=-\frac{1}{2} \int\left[\left(w^{1}\right)^{2}-\left(w^{2}\right)^{2}-\left(w^{3}\right)^{2}\right] d t
$$

The second Poisson structure (8.7) for the KdV system (8.3) is generated by the three-

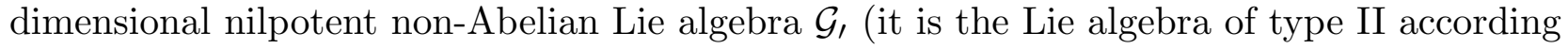
to the Bianchi classification of three-dimensional Lie algebras) and 2-cocycle on its loop algebra. Note that any nilpotent non-Abelian Lie algebra contains subalgebra isomorphic to $\mathcal{G}_{1}$.

Consider the special system of type (8.1):

$$
u_{t}^{i}=a^{i} u_{x}^{i}+u^{i} \sum_{k}\left(a^{i}-a^{k}\right) u^{k}
$$

where $a^{i} \neq a^{j}$, if $i \neq j, i, j=1, \ldots, N$.

Theorem 8.1. The system (8.8) is integrable and equivalent to some integrable homogeneous diagonal Hamiltonian system of hydrodynamic type by a combination of a reciprocal transformation and some changes of the fields and independent variables.

First of all, let us introduce new fields variables $w^{i}(x)$ by the relation

$$
u^{i}=e^{w^{i}}
$$

Then our system (8.8) will have the following form:

$$
w_{t}^{i}=a^{i} w_{x}^{i}+\sum_{k}\left(a^{i}-a^{k}\right) e^{w^{k}}
$$

Consider so-called reciprocal transformations of the system (8.9), or, in other words, transformations of the independent variables $x$ and $t$ in according to a solution $w(x, t)$ of the system (see also $[42,51]$ ):

$$
\begin{aligned}
d x^{\prime} & =\varphi_{1}(x, t, w) d x-\psi_{1}(x, t, w) d t \\
d t^{\prime} & =\varphi_{2}(x, t, w) d x-\psi_{2}(x, t, w) d t
\end{aligned}
$$

where

$$
\frac{\partial \varphi_{i}(x, t, w)}{\partial t}+\frac{\partial \psi_{i}(x, t, w)}{\partial x}=0, \quad i=1,2
$$




$$
\Delta=\varphi_{1} \psi_{2}-\varphi_{2} \psi_{1} \neq 0 .
$$

Here $w^{i}(x, t)$ is an arbitrary solution of the system (8.9), $\varphi_{i}, \psi_{i}, i=1,2$, are generated by some conservation laws (8.11) of the system (8.9).

Let us consider the following two conservation laws of the system (8.9):

1) $\varphi_{1}=-\sum_{k} e^{w^{k}}, \psi_{1}=\sum_{k} a^{k} e^{w^{k}}$

2) $\varphi_{2}=0, \psi_{2}=1$

Using the relations

$$
\begin{gathered}
w_{x}^{i}=w_{x^{\prime}}^{i} \varphi_{1}+w_{t^{\prime}}^{i} \varphi_{2}, \\
w_{t}^{i}=-w_{x^{\prime}}^{i} \psi_{1}-w_{t^{\prime}}^{i} \psi_{2},
\end{gathered}
$$

after corresponding reciprocal transformation (8.10)-(8.11) we obtain the following nonhomogeneous system of hydrodynamic type:

$$
w_{t^{\prime}}^{i}=\left(\sum_{k}\left(a^{i}-a^{k}\right) e^{w^{k}}\right)\left(w_{x^{\prime}}^{i}-1\right)
$$

After transformations

$$
v^{i}=w^{i}-x^{\prime}
$$

and

$$
x^{\prime \prime}=-e^{-x^{\prime}}
$$

we obtain diagonal weakly nonlinear semi-Hamiltonian homogeneous system of hydrodynamic type which is integrable due to the Tsarev theorem $[5,6]$ :

$$
v_{t^{\prime}}^{i}=\left(\sum_{k}\left(a^{i}-a^{k}\right) e^{v^{k}}\right) v_{x^{\prime \prime}}^{i}
$$

\section{Killing-Poisson bivectors on Riemannian manifolds and integrable bihamilto- nian hierarchies of Heisenberg magnet type}

The Killing-Poisson bivector $\omega^{i j}(u)$ on $N$-dimensional Riemannian manifold $\left(M, g_{i j}\right)$ is by definition (see [53]) a skew-symmetric tensor on $M\left(\omega^{i j}=-\omega^{j i}\right)$ which satisfies two the well-known relations: (1.1) (the Jacobi identity for the Poisson bivector $\omega^{i j}(u)$ ) and (7.15) (the identity for the Killing bivector $\omega^{i j}(u)$ on the Riemannian manifold $\left(M, g_{i j}\right)$ with the Levi-Civita connection $\nabla$ generated by the metric $g_{i j}$ ). It was shown in section 7 (see also [52]) that Killing-Poisson bivectors on the manifolds of constant Riemannian curvature $K$ define natural nonlocal (if $K \neq 0$ ) Poisson structures for nonhomogeneous systems of hydrodynamic type. Here we shall give complete description of the Killing-Poisson bivectors on the manifolds of constant Riemannian curvature in terms of Lie algebras with invariant scalar products and show that the compatible pairs of Poisson structures given by these bivectors generate also integrable bihamiltonian hierarchies of Heisenberg magnet type [52].

It is easy to describe all Killing-Poisson bivectors on flat spaces or, in other words, spaces of zero Riemannian curvature. In this case we shall consider flat coordinates $\left(v^{1}, \ldots, v^{N}\right)$ in 
which the metric $g_{i j}$ is constant. A tensor $\omega^{i j}(v)$ is a Killing-Poisson bivector on flat space $\left(M, g_{i j}\right)$ if and only if in flat coordinates $\left(v^{1}, \ldots, v^{N}\right)$ the following conditions are valid:

$$
\omega^{i j}(v)=c_{k}^{i j} v^{k}+d^{i j}
$$

where $c_{k}^{i j}, d^{i j}$ are constants such that

1) $c_{k}^{i j}$ are structural constants of a Lie algebra with invariant scalar product $<,>$ given by the metric $g_{i j}:<\operatorname{adX}(Y), Z>=-<Y, a d X(Z)>$;

2) $d^{i j}$ is a 2-cocycle on this Lie algebra:

$$
d^{i j}=-d^{j i}, \quad \sum_{(i, j, k)} c_{s}^{i j} d^{s k}=0
$$

where the sum is taken with respect to all cyclic permutations of the indices $i, j, k$.

For example, any semi-simple Lie algebra gives a Killing-Poisson bivector on flat space (we must take the Killing metric on the Lie algebra as a metric for corresponding flat space). This description coincides with classification of local nonhomogeneous Poisson brackets of hydrodynamic type $[3,4]$.

Let us consider arbitrary metric $g^{i j}$ of constant Riemannian curvature $K$ in canonical variables $u^{1}, \ldots, u^{N}$ :

$$
\begin{gathered}
\left(g^{i j}(u)\right)=(\lambda(u))^{2}\left(\begin{array}{cccc}
\varepsilon_{1} & 0 & \ldots & 0 \\
0 & \varepsilon_{2} & \ldots & 0 \\
\vdots & \vdots & \ddots & \vdots \\
0 & 0 & \ldots & \varepsilon_{N}
\end{array}\right) \\
\varepsilon_{i}= \pm 1, \quad \lambda(u)=1+\frac{K}{4} \sum_{i} \varepsilon_{i}\left(u^{i}\right)^{2}
\end{gathered}
$$

Theorem 9.1. For $N=2$ a tensor $\omega^{i j}(u)$ is a Killing-Poisson bivector on some space $\left(M, g_{i j}\right)$ of constant Riemannian curvature $K$ if and only if in canonical variables $\left(u^{1}, u^{2}\right)$

$$
\left(\omega^{i j}(u)\right)=c \lambda^{2}(u)\left(\begin{array}{cc}
0 & 1 \\
-1 & 0
\end{array}\right)
$$

where $c$ is an arbitrary constant.

As it was shown in [52] (see also section 7) any Killing-Poisson bivector $\omega^{i j}(u)$ on the space $\left(M, g_{i j}\right)$ of constant Riemannian curvature $K$ define pair of compatible Hamiltonian operators

$$
\begin{gathered}
M_{1}^{i j}=g^{i j}(u) \frac{d}{d x}-g^{i s}(u) \Gamma_{s k}^{j}(u) u_{x}^{k}+K u_{x}^{i}\left(\frac{d}{d x}\right)^{-1} u_{x}^{j}, \\
M_{2}^{i j}=\omega^{i j}(u),
\end{gathered}
$$

which generate the integrable hierarchy of the generalized $N$-component Heisenberg magnet equations

$$
S_{t}=\left[S, S_{x x}\right], \quad S^{2}=1,
$$

where $S$ is an $(N+1)$-vector and [, ] is the commutator in an $(N+1)$-dimensional Lie algebra equipped with an invariant inner product. The classic Heisenberg magnet corresponds to 
the simplest case of two-dimensional sphere $(N=2)$. For two-dimensional sphere $S^{1^{2}}+$ $S^{2}+S^{3^{2}}=1$ in coordinates of stereographic projection

$$
S^{1}=u^{1} / P, \quad S^{2}=u^{2} / P, \quad S^{3}=(P-1) / P,
$$

where $P=\left(u^{1^{2}}+u^{2^{2}}+1\right) / 2$, the metric has the form

$$
\left(g_{i j}\right)=\frac{1}{P^{2}}\left(\begin{array}{ll}
1 & 0 \\
0 & 1
\end{array}\right)
$$

The corresponding nonlocal Hamiltonian operator (9.3) generated by the metric has the form

$$
M_{1}=P^{2}\left(\begin{array}{cc}
d & 0 \\
0 & d
\end{array}\right)+P\left(\begin{array}{cc}
u^{1} u_{x}^{1}+u^{2} u_{x}^{2} & u^{1} u_{x}^{2}-u^{2} u_{x}^{1} \\
u^{2} u_{x}^{1}-u^{1} u_{x}^{2} & u^{1} u_{x}^{1}+u^{2} u_{x}^{2}
\end{array}\right)+\left(\begin{array}{cc}
u_{x}^{1} d^{-1} \circ u_{x}^{1} & u_{x}^{1} d^{-1} \circ u_{x}^{2} \\
u_{x}^{2} d^{-1} \circ u_{x}^{1} & u_{x}^{2} d^{-1} \circ u_{x}^{2}
\end{array}\right)
$$

In according to the Theorem 9.1 the unique (up to a constant factor) Killing-Poisson bivector on two-dimensional sphere in these coordinates has the form

$$
M_{2}=\left(\omega^{i j}(u)\right)=\left(\begin{array}{cc}
0 & -P^{2} \\
P^{2} & 0
\end{array}\right)
$$

Following to the well-known general construction of bihamiltonian equations (see $[37,61,62]$ ) consider the recursion operator $R=M_{1}\left(M_{2}\right)^{-1}$ corresponding to the compatible Hamiltonian pair $(9.5),(9.6)$ and apply it to the simplest translation flow

$$
\left(\begin{array}{l}
u^{1} \\
u^{2}
\end{array}\right)_{t}=\left(\begin{array}{l}
u^{1} \\
u^{2}
\end{array}\right)_{x}
$$

We obtain the new system

$$
\left(\begin{array}{l}
u^{1} \\
u^{2}
\end{array}\right)_{t}=R\left(\begin{array}{l}
u^{1} \\
u^{2}
\end{array}\right)_{x}
$$

or

$$
\left\{\begin{array}{l}
u_{t}^{1}=u_{x x}^{2}+\left(u^{2}\left(u_{x}^{1}\right)^{2}-2 u^{1} u_{x}^{1} u_{x}^{2}-u^{2}\left(u_{x}^{2}\right)^{2}\right) / P \\
u_{t}^{2}=-u_{x x}^{1}-\left(u^{1}\left(u_{x}^{2}\right)^{2}-2 u^{2} u_{x}^{1} u_{x}^{2}-u^{1}\left(u_{x}^{1}\right)^{2}\right) / P
\end{array}\right.
$$

which exactly coincides with the classic Heisenberg magnet equations

$$
\vec{S}_{t}=\vec{S} \times \vec{S}_{x x}, \quad \vec{S}^{2}=1
$$

Theorem 9.2 [52]. The compatible Hamiltonian pair (9.5),(9.6) generates the hierarchy of the Heisenberg magnet equations.

Explicit bihamiltonian representation of the Heisenberg magnet equations (9.7) has the form

$$
\left(\begin{array}{l}
u^{1} \\
u^{2}
\end{array}\right)_{t}=M_{1}\left(\begin{array}{l}
\delta G / \delta u^{1} \\
\delta G / \delta u^{2}
\end{array}\right)=M_{2}\left(\begin{array}{l}
\delta H / \delta u^{1} \\
\delta H / \delta u^{2}
\end{array}\right)
$$

with Hamiltonians

$$
G=\int \frac{u^{2} u_{x}^{1}-u^{1} u_{x}^{2}}{(2 P-1) P} d x, \quad H=\frac{1}{2} \int \frac{\left(u_{x}^{1}\right)^{2}+\left(u_{x}^{2}\right)^{2}}{P^{2}} d x
$$


Note that bihamiltonian representation of Heisenberg magnet equations was found in $[59,60]$ (in coordinates $S^{1}, S^{2}, S^{3}$ ).

Let us give a complete description of the Killing-Poisson bivectors on $N$-dimensional sphere $[52]$

$$
\sum_{k=1}^{N+1}\left(S^{k}\right)^{2}=1
$$

Let $c_{k}^{i j}$ be structural constants of some $(N+1)$-dimensional Lie algebra equipped with invariant scalar (Euclidean) product such that $c_{k}^{i j}+c_{i}^{k j}=0$. Consider the Lie-Poisson bivector $\Omega^{i j}=c_{k}^{i j} S^{k}$ and restrict it on the sphere (9.10). After restriction we obtain a Killing-Poisson bivector on $N$-dimensional sphere. The converse is also true.

Theorem 9.3 [52]. All Killing-Poisson bivectors on $N$-dimensional sphere can be obtained by the above construction from any Lie algebra with an invariant scalar product.

In coordinates $\left(u^{1}, \ldots, u^{N}\right)$ of the stereographic projection

$$
S^{1}=u^{1} / P, \ldots, S^{N}=u^{N} / P, S^{N+1}=(P-1) / P, \quad P=\left(\sum_{s=1}^{N}\left(u^{s}\right)^{2}+1\right) / 2
$$

we have

$$
\omega^{i j}=\left.\Omega\right|_{S^{N}}=P \sum_{s=1}^{N}\left(c_{N+1}^{s i} u^{s} u^{j}-c_{N+1}^{s j} u^{s} u^{i}+c_{s}^{i j} u^{s}\right)+c_{N+1}^{i j}(P-1) P
$$

For example, the Killing-Poisson bivector (9.6) on two-dimensional sphere is a result of restriction of the Lie-Poisson bivector

$$
\Omega=\left(\begin{array}{ccc}
0 & S^{3} & -S^{2} \\
-S^{3} & 0 & S^{1} \\
S^{2} & -S^{1} & 0
\end{array}\right)
$$

In order to obtain a complete description of the Killing-Poisson bivectors on arbitrary spaces of constant Riemannian curvature it is necessary to consider Lie algebras equipped with arbitrary invariant scalar products (with arbitrary signatures) and apply the same construction. The corresponding Killing-Poisson bivector gives a compatible Hamiltonian pair (9.3), (9.4) which generates a bihamiltonian integrable $N$-component system of Heisenberg magnet type.

\section{Nonlinear partial differential equations of associativity in $2 \mathrm{D}$ topological field theories and nondiagonalizable integrable systems of hydrodynamic type}

In this section we shall consider so-called nonlinear partial differential equations of associativity in 2D topological field theories (see [54-57]) and give their description as integrable nondiagonalizable weakly nonlinear systems of hydrodynamic type. For systems of this type corresponding general differential geometric theory of integrability connected with Poisson structures of hydrodynamic type can be developed. 
We remind very briefly following to Dubrovin [54] the basic mathematical concepts connected with the Witten-Dijkgraaf-E.Verlinde-H.Verlinde (WDVV) system arising originally in two-dimensional topological field theories $[56,57]$ and its relations with the Dubrovin type equations of associativity.

Consider a function $F(t), t=\left(t^{1}, \ldots, t^{N}\right)$ such that the following three conditions are satisfied for its third derivatives denoted as

$$
c_{\alpha \beta \gamma}(t)=\frac{\partial^{3} F(t)}{\partial t^{\alpha} \partial t^{\beta} \partial t^{\gamma}}:
$$

1) normalization, i.e.,

$$
\eta_{\alpha \beta}=c_{1 \alpha \beta}(t)
$$

is a constant nondegenerate matrix;

2) associativity, i.e., the functions

$$
c_{\alpha \beta}^{\gamma}(t)=\eta^{\gamma \epsilon} c_{\epsilon \alpha \beta}(t)
$$

for any $t$ define a structure of an associative algebra $A_{t}$ in the $\mathrm{N}$-dimensional space with a basis $e_{1}, \ldots, e_{N}$ :

$$
e_{\alpha} \cdot e_{\beta}=c_{\alpha \beta}^{\gamma}(t) e_{\gamma}
$$

3) $F(t)$ must be quasihomogeneous function of its variables:

$$
F\left(c^{d_{1}} t^{1}, \ldots, c^{d_{N}} t^{N}\right)=c^{d_{F}} F\left(t^{1}, \ldots, t^{N}\right)
$$

for any nonzero $c$ and for some numbers $d_{1}, \ldots, d_{N}, d_{F}$.

The resulting system of equations for $F(t)$ is called the Witten-Dijkgraaf-E.VerlindeH.Verlinde (WDVV) system [56,57] (see also [54-55]). It was shown by Dubrovin [54] that solutions of the WDVV system can be reduced by a linear change of coordinates to two special types:

(1) in the most important physically case

$$
F(t)=\frac{1}{2}\left(t^{1}\right)^{2} t^{N}+\frac{1}{2} t^{1} \sum_{\alpha=2}^{N-1} t^{\alpha} t^{N-\alpha+1}+f\left(t^{2}, \ldots, t^{N}\right)
$$

for some function $f\left(t^{2}, \ldots, t^{N}\right)$

(2) in some special case

$$
F(t)=\frac{c}{6}\left(t^{1}\right)^{3}+\frac{1}{2} t^{1} \sum_{\alpha=1}^{N-1} t^{\alpha} t^{N-\alpha+1}+f\left(t^{2}, \ldots, t^{N}\right)
$$

for a nonzero constant $c$.

If $N=3$ (first nontrivial case for the condition of associativity in algebra $A_{t}$ ) then for the first type solutions (10.1) of the WDVV system the associativity condition in algebra $A_{t}$ gives the following nonlinear partial differential equation for the function $f(x, y)$ [54]:

$$
f_{x x y}^{2}=f_{y y y}+f_{x x x} f_{x y y}
$$


Let us introduce new variables $a, b, c, d$ such that

$$
\begin{aligned}
& a=f_{x x x}, \quad b=f_{x x y}, \\
& c=f_{x y y}, \quad d=f_{y y y}
\end{aligned}
$$

Conditions of compatibility have the form:

$$
\left\{\begin{array}{l}
a_{y}=b_{x} \\
b_{y}=c_{x} \\
c_{y}=d_{x}
\end{array}\right.
$$

Besides, we have the relation

$$
d=b^{2}-a c
$$

from (10.3).

So, the equation (10.3) is equivalent to the following homogeneous system of hydrodynamic type

$$
\left(\begin{array}{l}
a \\
b \\
c
\end{array}\right)_{y}=\left(\begin{array}{ccc}
0 & 1 & 0 \\
0 & 0 & 1 \\
-c & 2 b & -a
\end{array}\right)\left(\begin{array}{l}
a \\
b \\
c
\end{array}\right)_{x}
$$

The system (10.7) is nondiagonalizable weakly nonlinear homogeneous system of hydrodynamic type. In fact, integrability of the system (10.7) follows from Dubrovin's results [55] but for the representation (10.7) it can be proved directly by usual Hamiltonian and differential geometric methods of hydrodynamic type systems.

Theorem 10.1 [58]. The equation (10.3) is equivalent to the integrable nondiagonalizable weakly nonlinear homogeneous system of hydrodynamic type (10.7).

Analogously, for $N=3$ and for the second type special solutions (10.2) of the WDVV system the associativity condition for algebra $A_{t}$ gives the following Dubrovin equation for the function $f(x, y)[54]$ :

$$
f_{x x x} f_{y y y}-f_{x x y} f_{x y y}=1
$$

After introducing new variables $a, b, c, d$ (10.4) from (10.8) we have the relation

$$
d=\frac{1+b c}{a}
$$

Compatibility conditions (10.5) and the relation (10.9) generate the following homogeneous system of hydrodynamic type

$$
\left(\begin{array}{l}
a \\
b \\
c
\end{array}\right)_{y}=\left(\begin{array}{ccc}
0 & 1 & 0 \\
0 & 0 & 1 \\
-\frac{(1+b c)}{a^{2}} & \frac{c}{a} & \frac{b}{a}
\end{array}\right)\left(\begin{array}{l}
a \\
b \\
c
\end{array}\right)_{x}
$$

Theorem 10.2 [58]. The equation (10.8) is equivalent to the integrable nondiagonalizable weakly nonlinear homogeneous system of hydrodynamic type (10.10). 
This work was partially supported by the Russian Foundation of Fundamental Researches (Grant No. 94-01-01478) and the International Science Foundation (Grant No. RKR000).

\section{References}

[1] B.A.Dubrovin and S.P.Novikov, Hydrodynamics of soliton lattices, Sov. Sci. Rev. C, Math. Phys. 9 (1993), part 4, 1-136.

[2] B.A.Dubrovin and S.P.Novikov, Hamiltonian formalizm of one-dimensional systems of hydrodynamic type and the Bogolyubov-Whitham averaging method, Dokl. Akad. Nauk SSSR, 270, No. 4 (1983) 781-785; (Soviet Math.Dokl., 27 (1983) 665-669).

[3] B.A.Dubrovin and S.P.Novikov, On Poisson brackets of hydrodynamic type, Dokl. Akad. Nauk SSSR, 279, No. 2 (1984) 294-297; (Soviet Math. Dokl., 30 (1984) 651$654)$.

[4] B.A.Dubrovin and S.P.Novikov, Hydrodynamics of weakly deformed soliton lattices. Differential geometry and Hamiltonian theory, Usp. Mat. Nauk, 44, No. 6 (1989) 29-98; (Russian Math. Surveys, 44, No. 6 (1989) 35-124).

[5] S.P.Tsarev, On Poisson brackets and one-dimensional Hamiltonian systems of hydrodynamic type, Dokl. Akad. Nauk SSSR, 282, No. 3 (1985) 534-537; (Soviet Math. Dokl., 31 (1985) 488-491).

[6] S.P.Tsarev, Geometry of Hamiltonian systems of hydrodynamic type. The generalized hodograph method, Izvestiya Akad. Nauk SSSR, Ser. Mat., 54, No. 5 (1990) 10481068; (Math. USSR Izvestiya, 37, No. 2 (1991) 397-419).

[7] O.I.Mokhov, Poisson brackets of Dubrovin-Novikov type (DN-brackets), Funkts. Analiz i ego Prilozh., 22, No. 4 (1988) 92-93; (Functional Anal. Appl., 22 (1988) 336-338).

[8] O.I.Mokhov, Symplectic forms on loop space and Riemannian geometry, Funkts. Analiz i ego Prilozh. 24, No.3 (1990), 86-87;(Functional Anal. Appl., 24 (1990)).

[9] O.I.Mokhov, Homogeneous second order symplectic structures on loop spaces and symplectic connections, Funkts. Analiz i ego Prilozh., 25, No. 2 (1991) 65-67; (Functional Anal. Appl., 25 (1991)).

[10] O.I.Mokhov, Canonical Hamiltonian representation of the Krichever-Novikov equation, Mat. Zametki, 50, No. 3 (1991) 87-96; (Math. Notes 939-945).

[11] O.I.Mokhov, Two-dimensional nonlinear sigma models and symplectic geometry on loop spaces of (pseudo)Riemannian manifolds, Nonlinear Evolution Equations and Dynamical Systems. Proceedings of the 8th Internat. Workshop (NEEDS'92), 6-17 July, 1992, Dubna, Russia, Ed. V.G.Makhan'kov, World Sci. Publishing, Singapore, 1993, 444-456

[12] I.Ya.Dorfman and O.I.Mokhov, Local symplectic operators and structures related to them, J. Math. Physics, 32, No. 12 (1991) 3288-3296

[13] O.I.Mokhov, Geometry of commuting differential operators of rank 3 and Hamiltonian flows, Ph.D. Thesis, Moscow State University, Moscow, Russia, 1984.

[14] O.I.Mokhov, Symplectic structures on loop spaces of smooth manifolds and Lagrangian systems of the field theory, Abstracts of the Internat. Geom. Colloquium, May 10-14, 1993, Moscow, Russia, 38-39 
[15] O.I.Mokhov and E.V.Ferapontov, On the nonlocal Hamiltonian hydrodynamic type operators connected with constant curvature metrics, Usp. Mat. Nauk, 45, No. 3 (1990) 191-192

[16] O.I.Mokhov, Hamiltonian systems of hydrodynamic type and constant curvature metrics, Phys. Letters, 166A, No. 3-4 (1992) 215-216

[17] E.V.Ferapontov, Differential geometry of nonlocal Hamiltonian operators of hydrodynamic type, Funkts. Analiz i ego Prilozh., 25, No. 3 (1991) 37-49

[18] S.P.Novikov, Andrejewski Lectures, Berlin, November-December 1993, Sfb 288 Preprint No. 117, 42 p.

[19] M.V.Pavlov, Multihamiltonian structures of the Whitham equations, Dokl. Akad. Nauk, 338, No. 2 (1994) 165-167

[20] V.V.Sokolov, On Hamiltonian property of the Krichever-Novikov equation, Dokl. Akad. Nauk SSSR, 272, No. 1 (1984) 48-50

[21] I.M.Krichever and S.P.Novikov, Holomorphic bundles and nonlinear equations. Finitezone solutions of rank 2, Dokl. Akad. Nauk SSSR, 247, No. 1 (1979) 33-37

[22] I.M.Krichever and S.P.Novikov, Holomorphic bundles over algebraic curves and nonlinear equations, Usp. Mat. Nauk, 35, No. 6 (1980) 47-68

[23] S.P.Novikov, Two-dimensional Schrödinger operators in periodic fields, Itogi Nauki i Tekhn., Seriya Sovremennye Problemy Matematiki, 23 (1983) 3-32

[24] O.I.Mokhov, Commuting ordinary differential operators of rank 3 corresponding to an elliptic curve, Usp. Mat. Nauk, 37, No. 4 (1982) 169-170

[25] O.I.Mokhov, Commuting differential operators of rank 3 and nonlinear equations, Izv. Akad. Nauk SSSR, Ser. Mat., 53, No. 6 (1989) 1291-1315

[26] S.I.Svinolupov and V.V.Sokolov, Evolution equations with nontrivial conservation laws, Funkts. Analiz i ego Prilozh., 16, No. 4 (1982) 86-87

[27] S.I.Svinolupov, V.V.Sokolov and R.I.Yamilov, Bäcklund transformations for integrable evolution equations, Dokl. Akad. Nauk SSSR, 271, No. 4 (1983) 802-805

[28] I.Ya.Dorfman, Krichever-Novikov equation and local symplectic structures, Dokl. Akad. Nauk SSSR, 302, No. 4 (1988) 792-795

[29] I.Ya.Dorfman, Dirac structures of integrable evolution equations, Phys. Letters, 125 A, No. 5 (1987) 240-246

[30] I.Ya.Dorfman, Dirac structures and integrability of nonlinear evolution equations, Wiley, England, 1993

[31] G.Wilson, On the quasi-hamiltonian formalism of the KdV equation, Phys.Letters, 132 A, No. 8-9 (1988) 445-450

[32] A.P.Fordy, in: Nonlinear Evolution Equations and Dynamical Systems, Proc. of the 8th Internat. Workshop (NEEDS'92), 6-17 July, 1992, Dubna, Russia, Ed. V.G.Makhan'kov, World Sci. Publishing, Singapore, 1993; M.Antonowicz and A.P.Fordy, Reports in Math. Physics, 1993

[33] G.Reeb, Comptes Rendus, 229, No. 20 (1949) 969-971

[34] G.Reeb, Bull. Cl. Sciences, Acad. Royale Belg., 5 Série, 36, No. 4 (1950) 324-329

[35] A.Weinstein, J. Diff. Geom., 9, No. 4 (1974) 513-517

[36] A.Besse, Manifolds with closed geodesics, Mir, Moscow, 1981

[37] F.Magri, A simple model of the integrable Hamiltonian equation, J. Math. Physics, 19, No. 5 (1978) 1156-1162 
[38] O.I.Mokhov, Symplectic geometry on loop spaces of smooth manifolds and nonlinear systems, Internat. Workshop "Theory of Nonlinear Waves", September 1991, Kaliningrad University, Kaliningrad, Russia

[39] O.I.Mokhov, Symplectic forms on loop spaces of Riemannian manifolds, Internat. Conference "Differential equations and related problems" in honour of 90-th anniversary of I.G.Petrovsky (1901-1973), May 1991, Moscow State University, Moscow, Russia

[40] O.I.Mokhov, Two-dimensional $\sigma$-models in the field theory: symplectic approach, Abstracts of the 9th Workshop "Modern Group Analysis. Methods and Applications", June 1992, Nizhniy Novgorod, Russia

[41] A.G.Meshkov, Hamiltonian and recursion operators for two-dimensional scalar fields, Phys. Letters, 170 A, No. 6 (1992) 405-408

[42] C.Rogers, Reciprocal transformations and their applications, in: Nonlinear Equations, Proc. 5th Workshop on Nonlinear Evolution Equations and Dynamical Systems (NEEDS'87), France, 1987, 109-123

[43] O.I.Mokhov and Y.Nutku, Bianchi transformation between the real hyperbolic MongeAmpère equation and the Born-Infeld equation, Letters in Math. Phys., 32, No. 2 (1994) 121-123

[44] G.V.Potemin, On Poisson brackets of differential-geometric type, Dokl. Akad. Nauk SSSR, 286, No. 1 (1986) 39-42; (Soviet Math. Dokl., 33 (1986) 30-33).

[45] G.V.Potemin, Ph.D. Thesis, Moscow State University, Moscow, Russia

[46] P.W.Doyle, Differential geometric Poisson bivectors in one space variable, J. Math. Phys., 34, No. 4 (1993) 1314-1338

[47] S.P.Tsarev, Mat. Zametki, 46, No. 1, (1989) 105-111

[48] O.I.Mokhov, On Hamiltonian structure of evolution with respect to the space variable $x$ for the Korteweg-de Vries equation, Usp. Mat. Nauk, 45, No. 1 (1990) 181-182

[49] O.I.Mokhov, Joint Hamiltonian representation of the Korteweg-de Vries equation and the three-wave equation, 1989

[50] Y.Nutku and Ö.Sarıo ğlu, An integrable family of Monge-Ampère equations and their multi-Hamiltonian structure, Phys. Letters, 173 A, No. 3 (1993) 270-274

[51] B.L.Rozhdestvensky, N.N.Yanenko, Systems of quasilinear equations and their applications to gas dynamics, Nauka, Moscow, 1978

[52] O.I.Mokhov and E.V.Ferapontov, Hamiltonian pairs associated with skew-symmetric Killing tensors on spaces of constant curvature, Funkts. Analiz i ego Prilozh., 28, No. 2 (1994) 60-63

[53] O.I.Mokhov, Killing-Poisson bivectors on Riemannian manifolds and integrable systems, Abstracts of Internat. Congress of Mathematicians, 3-11 August 1994, Zürich, Switzerland, 50

[54] B.A.Dubrovin, Geometry of 2D topological field theories, Preprint SISSA-89/94/FM, 1994, $204 \mathrm{p}$.

[55] B.A.Dubrovin, Integrable systems in topological field theory, Nucl. Physics B, 379 (1992) 627-689

[56] E.Witten, On the structure of the topological phase of two-dimensional gravity, Nucl. Physics B, 340 (1990) 281-332

[57] R.Dijkgraaf, E.Verlinde and H.Verlinde, Nucl. Physics B, 352 (1991) 59; Notes on topological string theory and 2D quantum gravity, Preprint PUPT-1217, IASSNSHEP-90/80, November, 1990 
[58] O.I.Mokhov, Differential equations of associativity in 2D topological field theories and geometry of nondiagonalizable systems of hydrodynamic type, Abstracts of Internat. Conference on Integrable Systems "Nonlinearity and Integrability: from Mathematics to Physics", February 21-24, 1995, Montpellier, France

[59] Yu.N.Sidorenko, Zap. Nauchn. Sem. LOMI, 161, No. 7 (1987) 76-87

[60] E.Barouch, A.S.Fokas and V.G.Papageorgiou, Journal of Math. Physics, 29, No. 12 (1988) 2628-2633

[61] I.M.Gelfand and I.Ya.Dorfman, Hamiltonian operators and algebraic structures related to them, Funkts. Analiz i ego Prilozh., 13, No. 4 (1979) 13-30; I.M.Gelfand and I.Ya.Dorfman, Schouten bracket and Hamiltonian operators, Funkts. Analiz i ego Prilozh., 14, No. 3 (1980) 71-74

[62] A.S.Fokas and B.Fuchssteiner, On the structure of symplectic operators and hereditary symmetries, Lettere al Nuovo Cimento, 28, No. 8 (1980) 299-303; B.Fuchssteiner and A.S.Fokas, Symplectic structures, their Bäcklund transformations and hereditary symmetries, Physica D, 4 (1981) 47-66

[63] I.Ya.Dorfman and F.W.Nijhoff, On a (2+1)-dimensional version of the Krichever-Novikov equation, Phys. Letters A, 157 (1991) 107-112 